\title{
Construction and validation of a 15-gene ferroptosis signature in lung adenocarcinoma
}

\author{
Guangxu Tu ${ }^{1,2}$, Weilin Peng ${ }^{1,2}$, Qidong Cai ${ }^{1,2}$, Zhenyu Zhao ${ }^{1,2}$, Xiong Peng ${ }^{1,2}$, Boxue He $^{1,2}$, Pengfei Zhang ${ }^{1,2}$, \\ Shuai Shi ${ }^{1,2}$, Yongguang Tao ${ }^{3,4}$, Xiang Wang ${ }^{\text {Corresp. 1,2 }}$ \\ 1 Department of Thoracic Surgery, The Second Xiangya Hospital, Central South University, Changsha, Hunan, China \\ 2 Hunan Key Laboratory of Early Diagnosis and Precision Therapy, Department of Thoracic Surgery, The Second Xiangya Hospital, Central South \\ University, Changsha, Hunan, China \\ 3 Key Laboratory of Carcinogenesis and Cancer Invasion, Ministry of Education, Department of Pathology, Xiangya Hospital, Central South University, \\ Changsha, Hunan, China \\ 4 NHC Key Laboratory of Carcinogenesis (Central South University), Cancer Research Institute and School of Basic Medicine, Central South University, \\ Changsha, Hunan, China \\ Corresponding Author: Xiang Wang \\ Email address: wangxiang@csu.edu.cn
}

Background: Ferroptosis is a novel form of programmed cell death characterized by the excessive accumulation of intracellular iron and an increase in reactive oxygen species. Emerging studies have shown that ferroptosis plays a vital role in the progression of lung adenocarcinoma, but the effect of ferroptosis-related genes on prognosis has been poorly studied. The purpose of this study was to explore the prognostic value of ferroptosisrelated genes. Methods: Lung adenocarcinoma samples were downloaded from The Cancer Genome Atlas (TCGA) and Gene Expression Omnibus (GEO) databases. The least absolute shrinkage and selection operator (LASSO) Cox regression algorithm was used to establish a predictive signature for risk stratification. Kaplan-Meier (K-M) survival analysis and receiver operating characteristic (ROC) curve analysis were conducted to evaluate the signature. We further explored the potential correlation between the risk score model and tumor immune status. Results: A 15-gene ferroptosis signature was constructed to classify patients into different risk groups. The overall survival (OS) of patients in the highrisk group was significantly shorter than that of patients in the low-risk group. The signature could predict OS independent of other risk factors. Single-sample gene set enrichment analysis (sSGSEA) identified the difference in immune status between the two groups. Patients in the high-risk group had stronger immune suppression, especially in the antigen presentation process. Conclusions: The 15-gene ferroptosis signature identified in this study could be a potential biomarker for prognosis prediction in lung adenocarcinoma. Targeting ferroptosis might be a promising therapeutic alternative for lung adenocarcinoma. 
1 Construction and validation of a 15-gene ferroptosis 2 signature in lung adenocarcinoma

3 Guangxu Tu${ }^{1,2}$, Weilin Peng ${ }^{1,2}$, Qidong Cai ${ }^{1,2}$, Zhenyu Zhao, ${ }^{1,2}$, Xiong Peng ${ }^{1,2}$, 4 Boxue $\mathrm{He}^{1,2}$, Pengfei Zhang ${ }^{1,2}$, Shuai Shi ${ }^{1,2}$, Yongguang Tao ${ }^{3,4}$, Xiang Wang ${ }^{1,2^{\star}}$

\section{*Corresponding Author:}

Xiang Wang

Address: 139 Renmin Road, Changsha, Hunan Province, 410011, China

Email address: wangxiang@csu.edu.cn

1 Department of Thoracic Surgery, The Second Xiangya Hospital, Central South University, Changsha, Hunan, China

2 Hunan Key Laboratory of Early Diagnosis and Precision Therapy, Department of Thoracic Surgery, The Second Xiangya Hospital, Central South University, Changsha, Hunan, China

3 Key Laboratory of Carcinogenesis and Cancer Invasion, Ministry of Education, Department of Pathology, Xiangya Hospital, Central South University, Changsha, Hunan, China

${ }^{4}$ NHC Key Laboratory of Carcinogenesis (Central South University), Cancer Research Institute and School of Basic Medicine, Central South University, Changsha, Hunan, China

\section{Abstract}

Background: Ferroptosis is a novel form of programmed cell death characterized by the excessive accumulation of intracellular iron and an increase in reactive oxygen species. Emerging studies have shown that ferroptosis plays a vital role in the progression of lung adenocarcinoma, but the effect of ferroptosis-related genes on prognosis has been poorly studied. The purpose of this study was to explore the prognostic value of ferroptosis-related genes.

Methods: Lung adenocarcinoma samples were downloaded from The Cancer Genome Atlas (TCGA) and Gene Expression Omnibus (GEO) databases. The least absolute shrinkage and selection operator (LASSO) Cox regression algorithm was used to establish a predictive signature for risk stratification. Kaplan-Meier (K-M) survival analysis and receiver operating characteristic $(R O C)$ curve analysis were conducted to evaluate the signature. We further explored the potential correlation between the risk score model and tumor immune status.

Results: A 15-gene ferroptosis signature was constructed to classify patients into different risk groups. The overall survival (OS) of patients in the high-risk group was significantly shorter than 
38 that of patients in the low-risk group. The signature could predict OS independent of other risk 39 factors. Single-sample gene set enrichment analysis (ssGSEA) identified the difference in 40 immune status between the two groups.

41 Conclusions: The 15-gene ferroptosis signature identified in this study could be a potential 42 biomarker for prognosis prediction in lung adenocarcinoma. Targeting ferroptosis might be a 43 promising therapeutic alternative for lung adenocarcinoma.

44

45

\section{Introduction}

46

47

48

49

50

51

52

53

54

55

56

57

58

59

60

61

62

63

64

65

66

67

68

Lung cancer has the highest morbidity and mortality worldwide (Bray et al. 2018). The most common subtype is lung adenocarcinoma (LUAD), accounting for approximately $40 \%$ of all lung cancers (Wei et al. 2018). Due to the lack of obvious clinical symptoms, most patients are diagnosed at relatively advanced stages and have 5-year survival rates of less than $15 \%$ (Denisenko et al. 2018). In recent years, targeted therapies, such as epidermal growth factor tyrosine kinase inhibitors (EGFR-TKIs), have achieved much success ( $\mathrm{Li}$ et al. 2016). However, drug resistance, which is an inevitable problem, causes the prognosis of LUAD patients to be far from satisfactory, and the survival rate at 5 years is still only $21 \%$ (Macheleidt et al. 2018). Therefore, it is meaningful to explore novel mechanisms of therapy and identify an effective prognostic model for risk stratification to improve the clinical outcomes of LUAD patients.

Iron is an indispensable element for human biological processes, while iron metabolism plays a dual role in the proliferation and death of tumor cells (Wang et al. 2019a). An increased level of iron within a limited range facilitates the proliferation of cancer cells, while the excessive accumulation of iron leads to the death of cancer cells by lipid peroxidation of the cell membrane, namely, ferroptosis (Mou et al. 2019; Stockwell et al. 2017). Ferroptosis is a novel form of regulated cell death and has a tumor suppressive function (Dixon et al. 2012). Emerging studies, though limited, have shown that ferroptosis plays a pivotal role in the regulation of tumor progression in non-small cell lung cancer (NSCLC). For example, SLC7A11, which could negatively regulate the process of ferroptosis, was reported to be overexpressed in LUAD and closely associated with tumor progression ( $\mathrm{Hu}$ et al. 2020; Ji et al. 2018; Ma et al. 2021). Upregulated GPX4 was reported to promote the proliferation of cancer cells and play a 
69 role in the resistance to ferroptosis in NSCLC ( $\mathrm{Ji}$ et al. 2018). High expression of NFS1 70 in LUAD could protect cancer cells from ferroptosis (Alvarez et al. 2017). High 71 expression of FSP1, EGLN1 and STRYK1 was found to be associated with greater 72 ferroptosis resistance in lung cancer cells (Doll et al. 2019; Jiang et al. 2017; Lai et al. 73 2019). In recent years, ferroptosis was reported to interact with some immune cells to 74 influence tumor progression. For example, abnormal ferroptosis-mediated cell death 75 could induce neutrophil recruitment and the inflammatory response to cancer cell death 76 (Pentimalli et al. 2019). Additionally, Wang et al. revealed that CD8+ T cells drive the 77 ferroptosis of cancer cells and enhance the antitumor effect, indicating a vital role of 78 ferroptosis in human anticancer immunity (Wang et al. 2019b; Zhang et al. 2020). All 79 these discoveries shed light on ferroptosis as a promising target for cancer therapy. 80 However, there are still limited studies exploring the potential role of ferroptosis-related 81 genes in LUAD so far, and their effect on prognosis remains largely unknown.

82 In this study, we comprehensively analyzed the expression patterns, prognostic value, 83 biological functions and potential pathways of ferroptosis-related genes to gain a better 84 85 86 understanding of ferroptosis in LUAD. Then, we constructed a prognostic signature of the ferroptosis-related genes in The Cancer Genome Atlas (TCGA) cohort and validated it in two Gene Expression Omnibus (GEO) datasets. Finally, we further explored the correlation between the prognostic signature and the immune status of LUAD patients. Our findings may provide useful information for further studies in this field.

\section{Methods}

\section{Acquisition of ferroptosis-related genes}

92 We downloaded the list of ferroptosis pathway genes (map04216) from the Kyoto 93 Encyclopedia of Genes and Genomes (KEGG) pathway database (https://www.genome.jp/kegg/pathway.html) and designated these genes as ferroptosisrelated genes. Iron metabolism-associated genes were retrieved from the R-HAS917937 pathway in the Reactome pathway database (https://reactome.org/) and the cellular iron ion homeostasis pathway in the AmiGO2 database (http://amigo.geneontology.org/amigo). After systematically searching and analyzing the original document, we discarded the genes that do not have a modulatory effect on 
100 ferroptosis. In addition, we collected and integrated newly reported ferroptosis-related 101 genes for subsequent research.

\section{Data collection}

103 We obtained the level 3 mRNA expression profiles and corresponding clinical data of 104 LUAD patients from the TCGA database (https://portal.gdc.cancer.gov/) and GEO 105 database (https://www.ncbi.nlm.nih.gov/geo/) (Rousseaux et al. 2013; Schabath et al.

106

107

108

109

110

111

112

113

114

115

116

117

118

119

120

121

122

123

124

125

126

127

128

129

130 2016) up to September 20, 2020. Samples with a follow-up time of less than 30 days or lack of prognostic data were excluded. Since the TCGA and GEO databases are public to researchers and we completely abided by the publication guidelines as well as the policies of access to the database, ethical review and approval were not required.

\section{Identification of differentially expressed ferroptosis genes}

The "limma" $\mathrm{R}$ package was used for the normalization of gene expression matrixes.

Then, we matched the mRNA sequencing data with the ferroptosis-related gene list and performed differential expression analysis between the tumor tissues and normal tissues in the TCGA cohort by using false discovery rate $(F D R)<0.05$ as the threshold. Thus, ferroptosis-related differentially expressed genes (DEGs) were identified. A heatmap and volcano plot to visualize the DEGs were generated by the "pheatmap" $R$ package. In addition, the protein-protein interaction (PPI) network of the ferroptosisrelated DEGs was analyzed in the Search Tool for the Retrieval of Interacting Genes (STRING) online database and visualized in Cytoscape 3.8.2 software. We also generated a correlation network of the DEGs using the "igraph" $R$ package.

\section{Establishment and validation of the prognostic model}

DEGs with prognostic value were screened by univariate Cox regression analysis with a $P$ value less than 0.05 . Then, the least absolute shrinkage and selection operator (LASSO) Cox regression algorithm was conducted to establish the signature. In brief, we used the normalized expression data as the independent variable and the overall survival (OS) data of the patients in the TCGA cohort as the response variable to perform the LASSO algorithm for the shrinkage of variables by the "glmnet" R package. Tenfold cross-validation was used to narrow the number of candidate genes and identify the penalty parameter $(\lambda)$, which corresponds to the lowest position of the likelihood deviance curve. We further evaluated the prognostic value of the LASSO genes by 
131 Kaplan-Meier (K-M) survival analysis. The K-M survival analysis was conducted using 132 the optimal cutoff determined by the "surv_cutpoint" function in the "survival" R package. 133 The risk score of each patient was calculated with the following formula: risk score $=\Sigma$ 134 (ExpmRNAn $\times \beta m R N A n)$. The patients were stratified by the median risk score into 135 high- and low-risk groups. After that, we performed principal component analysis (PCA) 136 to evaluate the discriminatory ability by the "status" $\mathrm{R}$ package. We evaluated the 137 predictive ability of the signature for OS through K-M survival analysis as well as time138 dependent receiver operating characteristic (ROC) curve analysis conducted by the 139 "survminer" and "survivalROC" R packages. Finally, we performed multivariate Cox regression analysis to identify independent risk factors for OS. In this study, the TCGA cohort was used as the derivation cohort, and the GSE72094 and GSE30219 cohorts were used as external validation cohorts.

\section{Construction and evaluation of the predictive nomogram}

144 We constructed a nomogram to predict the survival probabilities at 1 year, 2 years and 3 145 years in the TCGA cohort by integrating all the independent risk factors using the "rms" $146 \mathrm{R}$ package. The patients were stratified into different risk groups, and K-M survival 147 analysis was conducted to analyze the OS difference between the different risk groups. 148 Then, we calculated the $\mathrm{C}$ index and performed time-dependent ROC analysis to further 149 validate the prediction accuracy of the nomogram. Moreover, a calibration plot was used 150 to evaluate the consistency between the predicted survival probability and the real 151 observation. The GSE72094 cohort was used for the external validation of the 152 nomogram.

\section{Correlation with immune status}

154 Using the "GSVA" R package, we calculated the enrichment score of 16 immune-related 155 cells as well as 12 immune-related functions for each patient by single-sample gene set enrichment analysis (sSGSEA). In brief, using a set of genes that correspond to a particular immune cell or immune function, the enrichment score was calculated in the gene expression matrix through the ssGSEA algorithm, and the enrichment scores were normalized for subsequent analysis. Then, the enrichment scores for diverse immune cells and functions of patients in different risk groups were compared to illustrate the potential correlation between ferroptosis and immune status. The $\mathrm{R}$ script used for this 
162 part of the analysis is provided in the GitHub website

163 (https://github.com/guangxu0109/ssGSEA.git).

164 Functional enrichment analysis

165 To gain insight into the molecular mechanisms of these ferroptosis-related genes, we 166 performed Gene Ontology (GO) and KEGG enrichment analyses of the DEGs between 167 the high-risk group and low-risk group in the TCGA cohort, which were screened by the 168 thresholds of $\mid \log 2$ fold change $(F C) \mid \geq 1$ and FDR $<0.05$. GO enrichment analysis was 169 performed by the "clusterProfiler" R package, and KEGG enrichment analysis was 170 performed by gene set enrichment analysis (GSEA) using GSEA 4.0.2 software. The 171 pathways with a $P$ value less than 0.05 were considered to be significantly enriched.

\section{Statistical analysis}

173 All statistical analyses were conducted in R software version 4.0.2. The Mann-Whitney 174 test was conducted to compare gene expression levels between tumor tissues and 175 normal tissues as well as to compare the ssGSEA enrichment scores between different 176 risk groups. A $P$ value less than 0.05 (if not otherwise specified) was considered 177 statistically significant.

\section{Results}

\section{Twenty-two genes correlated with prognosis were identified in the TCGA cohort}

181 We generated a flowchart to describe the design of this study, as shown in Figure 1. A total of 182948 LUAD patients from the TCGA database $(\mathrm{N}=477)$, GSE72094 cohort $(\mathrm{N}=386)$ and 183 GSE30219 cohort ( $N=85)$ were used for subsequent analysis in this study. Table S1 shows the 184 basic characteristics of these patients. A total of 125 ferroptosis-associated genes (as listed in 185 Table S2) were identified to intersect with the mRNA expression matrix of the TCGA and GEO 186 databases. Ninety-seven genes were confirmed to be differentially expressed between tumor 187 tissues and nontumorous tissues (62 upregulated and 35 downregulated) in the TCGA cohort 188 (Figure 2A, B and Table S3). The PPI network and the correlation network of the DEGs are 189 shown in Figure S1. Twenty-two genes were identified to be correlated with prognosis through 190 univariate Cox regression analysis (Figure 3 ).

191 Establishment and assessment of the prognostic ferroptosis signature in the TCGA 192 cohort 
193 A signature consisting of 15 genes was established based on the minimum $\lambda$ value identified by 194 the LASSO algorithm in the TCGA cohort (Figure S2). The risk score of each patient was 195 calculated with the following formula: $\left(-0.037^{*}\right.$ expression level of AGER) + $(0.261$ * expression 196 level of CISD1 $)+(-0.019$ * expression level of DPP4 $)+(0.173$ * expression level of EGLN1) + $197(0.077$ * expression level of FANCD2 $)+(-0.253$ * expression level of GLS2) + $(-0.233$ * 198 expression level of ISCU $)+(0.087$ * expression level of ITGA6) + $(0.024$ * expression level of 199 ITGB4 $)+(0.118$ * expression level of KRAS $)+(0.109$ * expression level of NEDD4 $)+(-0.048$ * 200 expression level of PEBP1) + $\left(-0.133^{*}\right.$ expression level of SLC11A2) + $\left(0.013^{*}\right.$ expression level 201 of TFAP2A $)+(0.208$ * expression level of VDAC1). We further performed K-M survival analysis 202 for the 15 ferroptosis-related genes in the prognostic signature. The results confirmed that all 203 these genes were significantly correlated with OS (Figure S3). Among them, AGER, DPP4, 204 GLS2, ISCU, PEBP1 and SLC11A2 were identified to be protective factors for OS, while the 205 remaining factors (CISD1, EGLN1, FANCD2, ITGA6, ITGB4, KRAS, NEDD4, TFAP2A, and 206 VDAC1) were risk factors, which was consistent with the results of univariate Cox analysis, as 207 shown in Figure 3.

208 The patients in the TCGA cohort were divided into different risk groups by the median risk score 209 (Table S4). The PCA plot in Figure 4A shows that patients in different risk groups were clearly 210 distributed in two directions, indicating that the gene signature had good discriminatory power.

211 The K-M curve showed that the OS of patients in the high-risk group was significantly lower than 212 that of patients in the low-risk group (Figure 4B, C). In addition, OS was significantly decreased 213 with increasing risk scores (Figure 4D). The area under the curve (AUC) value of the risk score 214 model to predict OS was 0.775 , which was the highest among all the risk factors, indicating the 215 superior predictive ability of the prognostic signature (Figure 5E).

216 External validation of the ferroptosis-related gene signature

217 To further validate the robustness of the signature, we applied the 15-gene ferroptosis signature 218 to the GSE72094 and GSE30219 cohorts. The patients in the two cohorts were stratified into 219 high- and low-risk groups by the median risk score (Table S5 and Table S6). In the GSE72094 220 cohort, the PCA plot showed that patients in different risk groups were distributed in two 221 directions (Figure 5A). The $\mathrm{K}-\mathrm{M}$ curve indicated that patients in the high-risk group had 222 significantly shorter OS than those in the low-risk group, and OS was significantly decreased 223 with increasing risk scores (Figure 5B-D). The AUC value of the risk score model was 0.711 , 224 which was the highest among all the risk factors (Figure 5E). In the GSE30219 cohort, the PCA 225 plots showed that patients in different groups were distributed in two directions (Figure 5F). The 226 K-M survival curve confirmed a significantly worse OS for patients in the high-risk group than for 
227 those in the low-risk group (Figure 5G). OS significantly decreased with increasing risk scores

228 (Figure $5 \mathrm{H}, \mathrm{I})$. The AUC value of the risk score model was 0.877 , which was the highest among 229 all the risk factors (Figure 5J). All these results indicated that the 15-gene ferroptosis signature 230 had a robust predictive performance.

\section{Independent prognostic value of the ferroptosis-related gene signature}

232 To further assess the independent prognostic value of the 15-gene ferroptosis signature, we 233 performed univariate and multivariate Cox regression analyses on all characteristics, including 234 age, sex, and TNM stage, and the risk score model based on the ferroptosis signature in both 235 the derivation cohort and two validation cohorts. The results are presented in Table 1. In the 236 TCGA cohort, TNM stage and risk score model were identified to be independent prognostic 237 factors for OS. In the GSE72094 cohort, sex, TNM stage and risk score model were 238 independent prognostic factors for OS. The risk score model was the only independent 239 prognostic factor for OS in the GSE30219 cohort.

240 Given that the risk score model was not the only independent prognostic factor in the TCGA and 241 GSE72094 cohorts, we further performed subgroup survival analysis to verify whether the 15242 gene ferroptosis signature could be independent of other risk factors to predict prognosis. In the 243 TCGA cohort, patients in stage I-II and stage III-IV were stratified into high-risk and low-risk 244 groups by the median risk score. K-M curves showed that the OS of the high-risk group was 245 significantly worse than that of the low-risk group regardless of whether patients were stage I-II 246 or stage III-IV, indicating that the 15-gene ferroptosis signature could predict OS independent of 247 TNM stage (Figure 6A, B). Similarly, in the GSE72094 cohort, the OS of the high-risk group was 248 significantly worse than that of the low-risk group regardless of whether the patient was male or 249 female, indicating that the signature could predict OS independent of sex (Figure 6C, D). For the 250 TNM stage subgroup, the OS of patients in the high-risk group was significantly shorter than 251 that of patients in the low-risk group except for patients in stage III and stage IV, which was 252 likely due to the small sample size of patients in these groups (Figure 6E, F). Overall, the 15253 gene ferroptosis signature could predict OS independent of other clinical characteristics.

\section{Construction and assessment of the predictive nomogram}

255 Based on the independent risk factors identified in the TCGA cohort, we constructed a 256 nomogram model to predict the OS probabilities of patients at 1,2 and 3 years for risk 257 assessment and earlier intervention to improve patient survival time (as shown in Figure 7A). As 258 depicted in Figure 7B, a significantly poorer prognosis was observed in the high-risk group. The $259 \mathrm{C}$ index for the nomogram model to predict OS was 0.770 (95\% Cl: $0.724,0.816)$, indicating 260 that the nomogram model had a robust predictive accuracy. We further evaluated the 
261 discrimination and calibration of the nomogram by time-dependent ROC curves and calibration

262 plots for 1-year and 3-year OS. The 1-year and 3-year calibration plots indicated that the 263 predicted survival was highly consistent with the actual survival in the TCGA cohort (Figure 7D, 264 E). As shown in Figure 7H and Figure 7I, the AUC values of the nomogram for predicting 1-year 265 and 3-year OS were 0.812 and 0.757 , respectively. All the AUC values of the nomogram were 266 superior to those of other independent risk factors, indicating that the nomogram had a better 267 predictive performance.

268 The nomogram was further validated in the GSE72094 cohort, while TNM stage information was 269 not given in the GSE30219 cohort. The results showed that the OS of patients in the high-risk 270 group was significantly shorter than that of patients in the low-risk group (Figure 7C). The C 271 index of the nomogram model was $0.708(95 \% \mathrm{Cl}: 0.660,0.756)$. The calibration plots indicated 272 great consistency between the predicted survival rate and the real observation (Figure 7F, G). 273 The AUC values of the nomogram for predicting 1-year and 3-year OS were 0.735 and 0.758 , 274 respectively, which were also superior to those of all other independent risk factors (Figure 7J, $275 \mathrm{~K})$.

\section{Functional analysis of the 15-gene ferroptosis signature}

277 GO and KEGG enrichment analyses were performed to elucidate the possible biological 278 functions and pathways involved in the 15-gene ferroptosis signature. The results of GO 279 enrichment analysis indicated that the DEGs between the high-risk group and the low-risk group 280 were mainly enriched in pathways of the cell cycle and immune response, such as chromosome 281 segregation, mitotic nuclear division, mitotic sister chromatid segregation, antimicrobial humoral 282 response and humoral immune response (Figure 8A and Table S7). As shown in Figure 8B and 283 Table 2, the KEGG enrichment analysis revealed that the top 5 pathways enriched in the high284 risk group were the cell cycle, ubiquitin-mediated proteolysis, oocyte meiosis, homologous 285 recombination and p53 signaling pathways. The top 5 pathways enriched in the low-risk group 286 were the arachidonic acid metabolism, primary bile acid biosynthesis, alpha linolenic acid 287 metabolism, asthma, and intestinal immune network for IgA production pathways.

\section{Correlation with immune status}

289 Given that pathways of the immune response were significantly enriched in the 15-gene 290 ferroptosis signature, we further sought to investigate the correlation between immune status 291 and risk score of the ferroptosis signature. The enrichment results are presented in Tables S8292 10. Then, we analyzed the difference in the enrichment score of immune cells and immune 293 functions between the high-risk and low-risk groups. By comparing the enrichment results of the 294 three cohorts, we found that enrichment scores of activated dendritic cells (aDCs), dendritic 
295 cells (DCs), immature dendritic cells (iDCs), mast cells and neutrophils were significantly lower 296 in the high-risk group than in the low-risk group (Figure 9A, C, E). Regarding immune-related 297 functions, higher enrichment score of antigen-presenting cell (APC) inhibition was found in the 298 high-risk groups of all three cohorts, while a higher enrichment score of the type II IFN (IFN-Y) 299 response was found in the low-risk group (Figure 9B, D, F). All these results indicated a close 300 correlation between risk score of the ferroptosis signature and immune cells as well as immune 301 functions.

302

303 Discussion

304 Ferroptosis is a novel form of programmed cell death characterized by the excessive 305 accumulation of intracellular iron and an increase in reactive oxygen species (ROS) 306 (Dixon et al. 2012). Disturbances in iron hemostasis lead to excessive intracellular iron 307 accumulation and may induce ferroptosis (Bogdan et al. 2016). In recent years, this 308 unique pattern of cell death has been the focus of a large number of studies, and it is 309 well characterized as a promising therapeutic alternative for various cancer types 310 (Hassannia et al. 2019). However, there are still limited studies regarding the specific 311 role of ferroptosis in LUAD as well as its potential mechanism and pathways. The rapid 312 development of RNA sequencing and microarrays and large-scale public databases 313 provide an opportunity to obtain a better understanding of these ferroptosis-related 314 genes and to construct a reliable ferroptosis-based prognostic signature.

315 In the present study, we systematically analyzed the expression profile of ferroptosis316 related genes in LUAD, and we found that the majority of ferroptosis-related genes 317 (77.6\%, 97/125) were differentially expressed between LUAD tumor tissues and normal 318 tissues. Univariate Cox regression analysis showed that 22 genes were associated with 319 OS, and LASSO Cox regression finally identified 15 genes (AGER, CISD1, DPP4, 320 EGLN1, FANCD2, GLS2, ISCU, ITGA6, ITGB4, KRAS, NEDD4, PEBP1, SLC11A2, 321 TFAP2A, and VDAC1) to construct the ferroptosis-related gene signature. Their 322 modulatory effects on ferroptosis are summarized in Table 3. Among them, CISD1, 323 DPP4, EGLN1, FANCD2, ISCU, ITGA6, ITGB4, KRAS and NEDD4 negatively regulated 324 ferroptosis, while AGER, GLS2, PEBP1, SLC11A2 and VDAC1 positively regulated 325 ferroptosis. The depletion of AGER inhibited autophagy-dependent ferroptosis (Wen et 326 al. 2019). Low CISD1 expression could contribute to the ferroptosis of hepatocellular 
327 cancer cells by iron-mediated intramitochondrial lipid peroxidation (Yuan et al. 2016).

328 The accumulation of DPP4 could protect cells from ferroptosis by inhibiting lipid 329 peroxidation in human colorectal cancer (Xie et al. 2017). A previous study indicated 330 that EGLN1 could act as an oncogene by inducing LSH expression, which inhibits 331 ferroptosis in lung cancer (Jiang et al. 2017). FANCD2 was found to act as a ferroptosis 332 suppressor by decreasing lipid peroxidation (Song et al. 2016). Upregulation of GLS2 333 exhibited antitumor effects in gastric cancer by promoting ferroptosis (Niu et al. 2019). 334 ISCU, a mitochondrial protein, could regulate iron metabolism and increase the 335 expression of $\mathrm{GSH}$, thus significantly alleviating ferroptosis (Du et al. 2019). Integrin 336 subunit alpha 6 (ITGA6) and integrin subunit beta 4 (ITGB4) belong to the integrin 337 family, which mainly functions in cell adhesion. Overexpression of ITGA6 and ITGB4 338 attenuated the ferroptosis induced by erastin, while knockout of ITGA6 and ITGB4 promoted ferroptosis in breast cancer cells (Brown et al. 2017). Activated mutant KRAS could promote the expression of SCL7A11, thus inhibiting ferroptosis in lung adenocarcinoma (Hu et al. 2020). The depletion of NEDD4 was found to promote the 342 ferroptosis induced by erastin by limiting the degradation of VDAC2/3 in melanoma 343 (Yang et al. 2020). PEBP1, a scaffold protein kinase cascade inhibitor, was reported to 344 promote ferroptosis by enabling lipoxygenase (Wenzel et al. 2017). SLC11A2, also 345 called DMT1, is a major iron transporter and contributes to iron uptake in most cell 346 types, and its upregulation could trigger ferroptosis in acute myocardial infarction mice 347 (Song et al. 2020). TFAP2A was reported to negatively modulate ferroptosis by 348 activating the NRF2 signaling pathway (Huang et al. 2020). Inhibition of VDAC1 could 349 significantly alleviate ferroptosis and improve cell survival by decreasing the ROS level 350 in mitochondria (Nagakannan et al. 2019). Subsequent K-M survival analyses confirmed 351 the prognostic value of the 15 ferroptosis-related genes, indicating their potential role in 352 the initiation and progression of LUAD. Unsurprisingly, the risk scores of the 15-gene 353 ferroptosis signature were demonstrated to be significantly associated with the OS of 354 LUAD patients in both the TCGA cohort and two external validation cohorts. The 355 signature was independent of other clinical characteristics in all three cohorts. In 356 addition, the nomogram integrating the independent risk factors, including the risk score 357 model, exhibited high predictive value and may help clinicians make optimal clinical 
358 decisions to improve the OS rate of LUAD patients. These findings indicated the 359 important role of ferroptosis in the progression of LUAD and the possibility of the 360 ferroptosis signature as a biomarker for OS.

361 KEGG pathway analysis showed that the high-risk group was mainly enriched in 362 pathways closely associated with tumorigenesis, such as the cell cycle, oocyte meiosis 363 and homologous recombination. GO analysis of the DEGs between the high-risk group 364 and low-risk group showed that the biological functions of the DEGs were mainly 365 enriched in the regulation of the cell cycle and immune response, indicating a link 366 between ferroptosis and antitumor immunity. Cancer growth and metastasis are closely 367 related to interactions with the immune system (Matsushita et al. 2012). When investigating the correlation with immune status, we interestingly found that enrichment 369 scores of aDCs, DCs, iDCs, mast cells and neutrophils were significantly lower in the 370 high-risk group. Among these differentially enriched immune cells, iDCs specialize in 371 antigen capture, and DCs are professional APCs (Lin et al. 2019; Tiberio et al. 2018).

372 Both aDCs and iDCs play crucial roles in the process of cytotoxic T cell activation and in 373 the regulation of the immune response to cancer cells (Durai \& Murphy 2016). 374 Moreover, neutrophils infiltrating tumor tissues, termed tumor-associated neutrophils 375 (TANs), also play a role in antitumor immunity (Lecot et al. 2019). It was confirmed that 376 TANs could release cytotoxic substances such as ROX, thus directly inducing tumor cell 377 apoptosis (Takeshima et al. 2016). The role of mast cells in the progression of tumors is 378 controversial. For instance, mast cells could play a protumorigenic role by releasing 379 angiogenic and lymphangiogenic factors, which promote angiogenesis and 380 lymphogenesis (Detoraki et al. 2009). In contrast, mast cells could secrete tumor 381 necrosis factor $\alpha(T N F-\alpha)$ and directly mediate tumor cell cytotoxicity, thus playing an 382 antitumorigenic role (Varricchi et al. 2017). In lung cancer, Bao et al. and Welsh et al. 383 reported that the low abundance of mast cell in cancer specimens was correlated with 384 worse OS (Bao et al. 2020; Welsh et al. 2005). When we analyzed the difference in 385 immune functions, a stronger inhibition of APCs was found in the high-risk groups of 386 both the derivation cohort and two validation cohorts. The synergistic effect of reduced 387 DC and stronger inhibition of APCs in the high-risk group may contribute to the great 388 suppression of the tumor antigen presentation process. Additionally, we also found that 
389

390

391

392

393

394

395

396

397

\section{8}

399

400

401

402

403

404

405

406

407

408

409

410

411

412

413

414

415

416

417

418

419

420

421

422

423

424

enrichment score of type II IFN was significantly lower in the high-risk group. IFN-Y produced by APCs, T cells, B cells, NK cells and NKT cells is the only member of the type II INF family (Castro et al. 2018). It is well known that IFN-y plays a pivotal role in cancer immune surveillance, stimulating antitumor immunity and facilitating the recognition and elimination of cancer cells (Benci et al. 2019; Shankaran et al. 2001; Street et al. 2001). All these findings suggested that ferroptosis may be involved in antitumor immune response. However, more experimental investigation is warranted to confirm these findings and reveal the underlying mechanism.

\section{Conclusions}

The 15-gene ferroptosis signature identified in this study could be a potential biomarker for prognosis prediction in LUAD. Targeting ferroptosis might be a promising therapeutic alternative for LUAD.

\section{Acknowledgments}

The authors are truly thankful to the TCGA and GEO databases for the availability of the data.

\section{References}

Alvarez SW, Sviderskiy VO, Terzi EM, Papagiannakopoulos T, Moreira AL, Adams S, Sabatini DM, Birsoy K, and Possemato R. 2017. NFS1 undergoes positive selection in lung tumours and protects cells from ferroptosis. Nature 551:639-643. 10.1038/nature24637

Bao X, Shi R, Zhao T, and Wang Y. 2020. Mast cell-based molecular subtypes and signature associated with clinical outcome in early-stage lung adenocarcinoma. Molecular oncology 14:917-932. 10.1002/1878-0261.12670

Benci JL, Johnson LR, Choa R, Xu Y, Quu J, Zhou Z, Xu B, Ye D, Nathanson KL, June CH, Wherry EJ, Zhang NR, Ishwaran H, Hellmann MD, Wolchok JD, Kambayashi T, and Minn AJ. 2019. Opposing Functions of Interferon Coordinate Adaptive and Innate Immune Responses to Cancer Immune Checkpoint Blockade. Cell 178. 10.1016/j.cell.2019.07.019

Bogdan AR, Miyazawa M, Hashimoto K, and Tsuji Y. 2016. Regulators of Iron Homeostasis: New Players in Metabolism, Cell Death, and Disease. Trends Biochem Sci 41:274-286. 10.1016/j.tibs.2015.11.012 
425

426

427

428

429

430

431

432

433

434

435

436

437

438

439

440

441

442

443

444

445

446

447

448

449

450

451

452

453

454

455

456

457

458

459

460

461

462

463

464

465

466

467

468

469

470

471

472

473

Bray F, Ferlay J, Soerjomataram I, Siegel RL, Torre LA, and Jemal A. 2018. Global cancer statistics 2018: GLOBOCAN estimates of incidence and mortality worldwide for 36 cancers in 185 countries. CA Cancer J Clin 68:394-424. 10.3322/caac.21492

Brown CW, Amante JJ, Goel HL, and Mercurio AM. 2017. The a6ß4 integrin promotes resistance to ferroptosis. Journal of Cell Biology 216:4287-4297. 10.1083/jcb.201701136 $\% J$ Journal of Cell Biology

Castro F, Cardoso AP, Gonçalves RM, Serre K, and Oliveira MJ. 2018. Interferon-Gamma at the Crossroads of Tumor Immune Surveillance or Evasion. Frontiers in immunology 9:847. 10.3389/fimmu.2018.00847

Denisenko TV, Budkevich IN, and Zhivotovsky B. 2018. Cell death-based treatment of lung adenocarcinoma. Cell Death Dis 9:117. 10.1038/s41419-017-0063-y

Detoraki A, Staiano RI, Granata F, Giannattasio G, Prevete N, de Paulis A, Ribatti D, Genovese A, Triggiani M, and Marone G. 2009. Vascular endothelial growth factors synthesized by human lung mast cells exert angiogenic effects. The Journal of allergy and clinical immunology 123. 10.1016/j.jaci.2009.01.044

Dixon SJ, Lemberg KM, Lamprecht MR, Skouta R, Zaitsev EM, Gleason CE, Patel DN, Bauer AJ, Cantley AM, Yang WS, Morrison B, and Stockwell BR. 2012. Ferroptosis: an irondependent form of nonapoptotic cell death. Cell 149:1060-1072. 10.1016/j.cell.2012.03.042

Doll S, Freitas FP, Shah R, Aldrovandi M, da Silva MC, Ingold I, Goya Grocin A, Xavier da Silva TN, Panzilius E, Scheel CH, Mourão A, Buday K, Sato M, Wanninger J, Vignane T, Mohana V, Rehberg M, Flatley A, Schepers A, Kurz A, White D, Sauer M, Sattler M, Tate EW, Schmitz W, Schulze A, O'Donnell V, Proneth B, Popowicz GM, Pratt DA, Angeli JPF, and Conrad M. 2019. FSP1 is a glutathione-independent ferroptosis suppressor. Nature 575:693-698. 10.1038/s41586-019-1707-0

Du J, Wang T, Li Y, Zhou Y, Wang X, Yu X, Ren X, An Y, Wu Y, Sun W, Fan W, Zhu Q, Wang $Y$, and Tong $X$. 2019. DHA inhibits proliferation and induces ferroptosis of leukemia cells through autophagy dependent degradation of ferritin. Free radical biology \& medicine 131:356-369. 10.1016/j.freeradbiomed.2018.12.011

Durai V, and Murphy KM. 2016. Functions of Murine Dendritic Cells. Immunity 45:719-736. 10.1016/j.immuni.2016.10.010

Hassannia B, Vandenabeele P, and Vanden Berghe T. 2019. Targeting Ferroptosis to Iron Out Cancer. Cancer cell 35:830-849. 10.1016/j.ccell.2019.04.002

Hu K, Li K, Lv J, Feng J, Chen J, Wu H, Cheng F, Jiang W, Wang J, Pei H, Chiao PJ, Cai Z, Chen Y, Liu M, and Pang X. 2020. Suppression of the SLC7A11/glutathione axis causes synthetic lethality in KRAS-mutant lung adenocarcinoma. The Journal of clinical investigation 130:1752-1766. 10.1172/JCl124049

Huang HX, Yang G, Yang Y, Yan J, Tang XY, and Pan Q. 2020. TFAP2A is a novel regulator that modulates ferroptosis in gallbladder carcinoma cells via the Nrf2 signalling axis. European review for medical and pharmacological sciences 24:4745-4755. 10.26355/eurrev_202005_21163

Ji X, Qian J, Rahman SM̄J, Siska PJ, Zou Y, Harris BK, Hoeksema MD, Trenary IA, Heidi C, Eisenberg R, Rathmell JC, Young JD, and Massion PP. 2018. xCT (SLC7A11)-mediated metabolic reprogramming promotes non-small cell lung cancer progression. Oncogene 37:5007-5019. 10.1038/s41388-018-0307-z

Jiang Y, Mao C, Yang R, Yan B, Shi Y, Liu X, Lai W, Liu Y, Wang X, Xiao D, Zhou H, Cheng Y, Yu F, Cao Y, Liu S, Yan Q, and Tao Y. 2017. EGLN1/c-Myc Induced Lymphoid-Specific Helicase Inhibits Ferroptosis through Lipid Metabolic Gene Expression Changes. Theranostics 7:3293-3305. 10.7150/thno.19988

Peer] reviewing PDF | (2021:01:57561:2:0:NEW 26 May 2021) 
474

475

476

477

478

479

480

481

482

483

484

485

486

487

488

489

490

491

492

493

494

495

496

497

498

499

500

501

502

503

504

505

506

507

508

509

510

511

512

513

514

515

516

517

518

519

520

521

522

523

524

Lai Y, Zhang Z, Li J, Li W, Huang Z, Zhang C, Li X, and Zhao J. 2019. STYK1/NOK correlates with ferroptosis in non-small cell lung carcinoma. Biochemical and biophysical research communications 519:659-666. 10.1016/j.bbrc.2019.09.032

Lecot P, Sarabi M, Pereira Abrantes M, Mussard J, Koenderman L, Caux C, Bendriss-Vermare N, and Michallet M-C. 2019. Neutrophil Heterogeneity in Cancer: From Biology to Therapies. Frontiers in immunology 10:2155-2155. 10.3389/fimmu.2019.02155

Li S, Choi Y-L, Gong Z, Liu X, Lira M, Kan Z, Oh E, Wang J, Ting JC, Ye X, Reinhart C, Liu X, Pei Y, Zhou W, Chen R, Fu S, Jin G, Jiang A, Fernandez J, Hardwick J, Kang MW, I H, Zheng H, Kim J, and Mao M. 2016. Comprehensive Characterization of Oncogenic Drivers in Asian Lung Adenocarcinoma. Journal of thoracic oncology : official publication of the International Association for the Study of Lung Cancer 11:2129-2140. 10.1016/j.jtho.2016.08.142

Lin C-L, Huang H-M, Hsieh C-L, Fan C-K, and Lee Y-L. 2019. Jagged1-expressing adenovirusinfected dendritic cells induce expansion of Foxp3(+) regulatory $\mathrm{T}$ cells and alleviate $\mathrm{T}$ helper type 2-mediated allergic asthma in mice. Immunology 156:199-212. 10.1111/imm.13021

Ma L, Chen T, Zhang X, Miao Y, Tian X, Yu K, Xu X, Niu Y, Guo S, Zhang C, Qiu S, Qiao Y, Fang $W$, Du L, Yu Y, and Wang J. 2021. The m(6)A reader YTHDC2 inhibits lung adenocarcinoma tumorigenesis by suppressing SLC7A11-dependent antioxidant function. Redox biology 38:101801-101801. 10.1016/j.redox.2020.101801

Macheleidt IF, Dalvi PS, Lim SY, Meemboor S, Meder L, Kasgen O, Muller M, Kleemann K, Wang L, Nurnberg P, Russeler V, Schafer SC, Mahabir E, Buttner R, and Odenthal M. 2018. Preclinical studies reveal that LSD1 inhibition results in tumor growth arrest in lung adenocarcinoma independently of driver mutations. Mol Oncol 12:1965-1979. 10.1002/1878-0261.12382

Matsushita H, Vesely MD, Koboldt DC, Rickert CG, Uppaluri R, Magrini VJ, Arthur CD, White JM, Chen Y-S, Shea LK, Hundal J, Wendl MC, Demeter R, Wylie T, Allison JP, Smyth MJ, Old LJ, Mardis ER, and Schreiber RD. 2012. Cancer exome analysis reveals a Tcell-dependent mechanism of cancer immunoediting. Nature 482:400-404. 10.1038/nature10755

Mou Y, Wang J, Wu J, He D, Zhang C, Duan C, and Li B. 2019. Ferroptosis, a new form of cell death: opportunities and challenges in cancer. J Hematol Oncol 12:34. 10.1186/s13045019-0720-y

Nagakannan P, Islam MI, Karimi-Abdolrezaee S, and Eftekharpour E. 2019. Inhibition of VDAC1 Protects Against Glutamate-Induced Oxytosis and Mitochondrial Fragmentation in Hippocampal HT22 Cells. Cellular and molecular neurobiology 39:73-85. 10.1007/s10571-018-0634-1

Niu Y, Zhang J, Tong Y, Li J, and Liu B. 2019. Physcion 8-O- $\beta$-glucopyranoside induced ferroptosis via regulating miR-103a-3p/GLS2 axis in gastric cancer. Life sciences 237:116893. 10.1016/j.Ifs.2019.116893

Pentimalli F, Grelli S, Di Daniele N, Melino G, and Amelio I. 2019. Cell death pathologies: targeting death pathways and the immune system for cancer therapy. Genes and immunity 20:539-554. 10.1038/s41435-018-0052-x

Rousseaux S, Debernardi A, Jacquiau B, Vitte A-L, Vesin A, Nagy-Mignotte H, Moro-Sibilot D, Brichon P-Y, Lantuejoul S, Hainaut P, Laffaire J, de Reyniès A, Beer DG, Timsit J-F, Brambilla C, Brambilla E, and Khochbin S. 2013. Ectopic activation of germline and placental genes identifies aggressive metastasis-prone lung cancers. Science translational medicine 5:186ra166. 10.1126/scitransImed.3005723

Schabath MB, Welsh EA, Fulp WJ, Chen L, Teer JK, Thompson ZJ, Engel BE, Xie M, Berglund AE, Creelan BC, Antonia SJ, Gray JE, Eschrich SA, Chen DT, Cress WD, Haura EB, and Beg AA. 2016. Differential association of STK11 and TP53 with KRAS mutation-

PeerJ reviewing PDF | (2021:01:57561:2:0:NEW 26 May 2021) 
525

526

527

528

529

530

531

532

533

534

535

536

537

538

539

540

541

542

543

544

545

546

547

548

549

550

551

552

553

554

555

556

557

558

559

560

561

562

563

564

565

566

567

568

569

570

571

572

573

574

associated gene expression, proliferation and immune surveillance in lung adenocarcinoma. Oncogene 35:3209-3216. 10.1038/onc.2015.375

Shankaran V, Ikeda H, Bruce AT, White JM, Swanson PE, Old LJ, and Schreiber RD. 2001. IFNgamma and lymphocytes prevent primary tumour development and shape tumour immunogenicity. Nature 410:1107-1111.

Song X, Xie Y, Kang R, Hou W, Sun X, Epperly MW, Greenberger JS, and Tang D. 2016. FANCD2 protects against bone marrow injury from ferroptosis. Biochem Biophys Res Commun 480:443-449. 10.1016/j.bbrc.2016.10.068

Song Y, Wang B, Zhu X, Hu J, Sun J, Xuan J, and Ge Z. 2020. Human umbilical cord bloodderived MSCs exosome attenuate myocardial injury by inhibiting ferroptosis in acute myocardial infarction mice. Cell biology and toxicology. 10.1007/s10565-020-09530-8

Stockwell BR, Friedmann Angeli JP, Bayir H, Bush Al, Conrad M, Dixon SJ, Fulda S, Gascón S, Hatzios SK, Kagan VE, Noel K, Jiang X, Linkermann A, Murphy ME, Overholtzer M, Oyagi A, Pagnussat GC, Park J, Ran Q, Rosenfeld CS, Salnikow K, Tang D, Torti FM, Torti SV, Toyokuni S, Woerpel KA, and Zhang DD. 2017. Ferroptosis: A Regulated Cell Death Nexus Linking Metabolism, Redox Biology, and Disease. Cell 171:273-285. 10.1016/j.cell.2017.09.021

Street SE, Cretney E, and Smyth MJ. 2001. Perforin and interferon-gamma activities independently control tumor initiation, growth, and metastasis. Blood 97:192-197.

Takeshima T, Pop LM, Laine A, lyengar P, Vitetta ES, and Hannan R. 2016. Key role for neutrophils in radiation-induced antitumor immune responses: Potentiation with G-CSF. Proceedings of the National Academy of Sciences of the United States of America 113:11300-11305.

Tiberio L, Del Prete A, Schioppa T, Sozio F, Bosisio D, and Sozzani S. 2018. Chemokine and chemotactic signals in dendritic cell migration. Cellular \& molecular immunology 15:346352. 10.1038/s41423-018-0005-3

Varricchi G, Galdiero MR, Loffredo S, Marone G, lannone R, Marone G, and Granata F. 2017. Are Mast Cells MASTers in Cancer? Frontiers in immunology 8:424-424. 10.3389/fimmu.2017.00424

Wang F, Lv H, Zhao B, Zhou L, Wang S, Luo J, Liu J, and Shang P. 2019a. Iron and leukemia: new insights for future treatments. Journal of experimental \& clinical cancer research : CR 38:406. 10.1186/s13046-019-1397-3

Wang W, Green M, Choi JE, Gijon M, Kennedy PD, Johnson JK, Liao P, Lang X, Kryczek I, Sell A, Xia H, Zhou J, Li G, Li J, Li W, Wei S, Vatan L, Zhang H, Szeliga W, Gu W, Liu R, Lawrence TS, Lamb C, Tanno Y, Cieslik M, Stone E, Georgiou G, Chan TA, Chinnaiyan A, and Zou W. 2019b. CD8(+) T cells regulate tumour ferroptosis during cancer immunotherapy. Nature 569:270-274. 10.1038/s41586-019-1170-y

Wei X, Zhang K, Qin H, Zhu J, Qin Q, Yu Y, and Wang H. 2018. GMDS knockdown impairs cell proliferation and survival in human lung adenocarcinoma. BMC cancer 18:600-600. 10.1186/s12885-018-4524-1

Welsh TJ, Green RH, Richardson D, Waller DA, O'Byrne KJ, and Bradding P. 2005. Macrophage and mast-cell invasion of tumor cell islets confers a marked survival advantage in non-small-cell lung cancer. Journal of clinical oncology : official journal of the American Society of Clinical Oncology 23:8959-8967.

Wen Q, Liu J, Kang R, Zhou B, and Tang D. 2019. The release and activity of HMGB1 in ferroptosis. Biochem Biophys Res Commun 510:278-283. 10.1016/j.bbrc.2019.01.090

Wenzel SE, Tyurina YY, Zhao J, St Croix CM, Dar HH, Mao G, Tyurin VA, Anthonymuthu TS, Kapralov AA, Amoscato AA, Mikulska-Ruminska K, Shrivastava IH, Kenny EM, Yang Q, Rosenbaum JC, Sparvero LJ, Emlet DR, Wen X, Minami Y, Qu F, Watkins SC, Holman TR, VanDemark AP, Kellum JA, Bahar I, Bayır H, and Kagan VE. 2017. PEBP1

Peer) reviewing PDF | (2021:01:57561:2:0:NEW 26 May 2021) 
Wardens Ferroptosis by Enabling Lipoxygenase Generation of Lipid Death Signals. Cell 171. 10.1016/j.cell.2017.09.044

576

577

578

579

580

581

582

583

584

585

586

587

588

589

590

591

Xie Y, Zhu S, Song X, Sun X, Fan Y, Liu J, Zhong M, Yuan H, Zhang L, Billiar TR, Lotze MT, Zeh HJ, Kang R, Kroemer G, and Tang D. 2017. The Tumor Suppressor p53 Limits Ferroptosis by Blocking DPP4 Activity. Cell reports 20:1692-1704. 10.1016/j.celrep.2017.07.055

Yang Y, Luo M, Zhang K, Zhang J, Gao T, Connell DO, Yao F, Mu C, Cai B, Shang Y, and Chen W. 2020. Nedd4 ubiquitylates VDAC2/3 to suppress erastin-induced ferroptosis in melanoma. Nature communications 11:433. 10.1038/s41467-020-14324-X

Yuan H, Li X, Zhang X, Kang R, and Tang D. 2016. CISD1 inhibits ferroptosis by protection against mitochondrial lipid peroxidation. Biochemical and biophysical research communications 478:838-844. 10.1016/j.bbrc.2016.08.034

Zhang Y, Song J, Zhao Z, Yang M, Chen M, Liu C, Ji J, and Zhu D. 2020. Single-cell transcriptome analysis reveals tumor immune microenvironment heterogenicity and granulocytes enrichment in colorectal cancer liver metastases. Cancer Letters 470:8494. 
Figure 1

Work flow of the study.

The TCGA cohort was used to construct the prognostic ferroptosis-related gene signature.

The GSE72094 cohort and GSE30219 cohort were used to further validate the prognostic signature. TCGA, The Cancer Genome Atlas; LUAD, lung adenocarcinoma; DEGs, differentially expressed genes; FDR, false discovery rate; OS, overall survival; PCA, principal component analysis; ROC, the receiver operating characteristic.

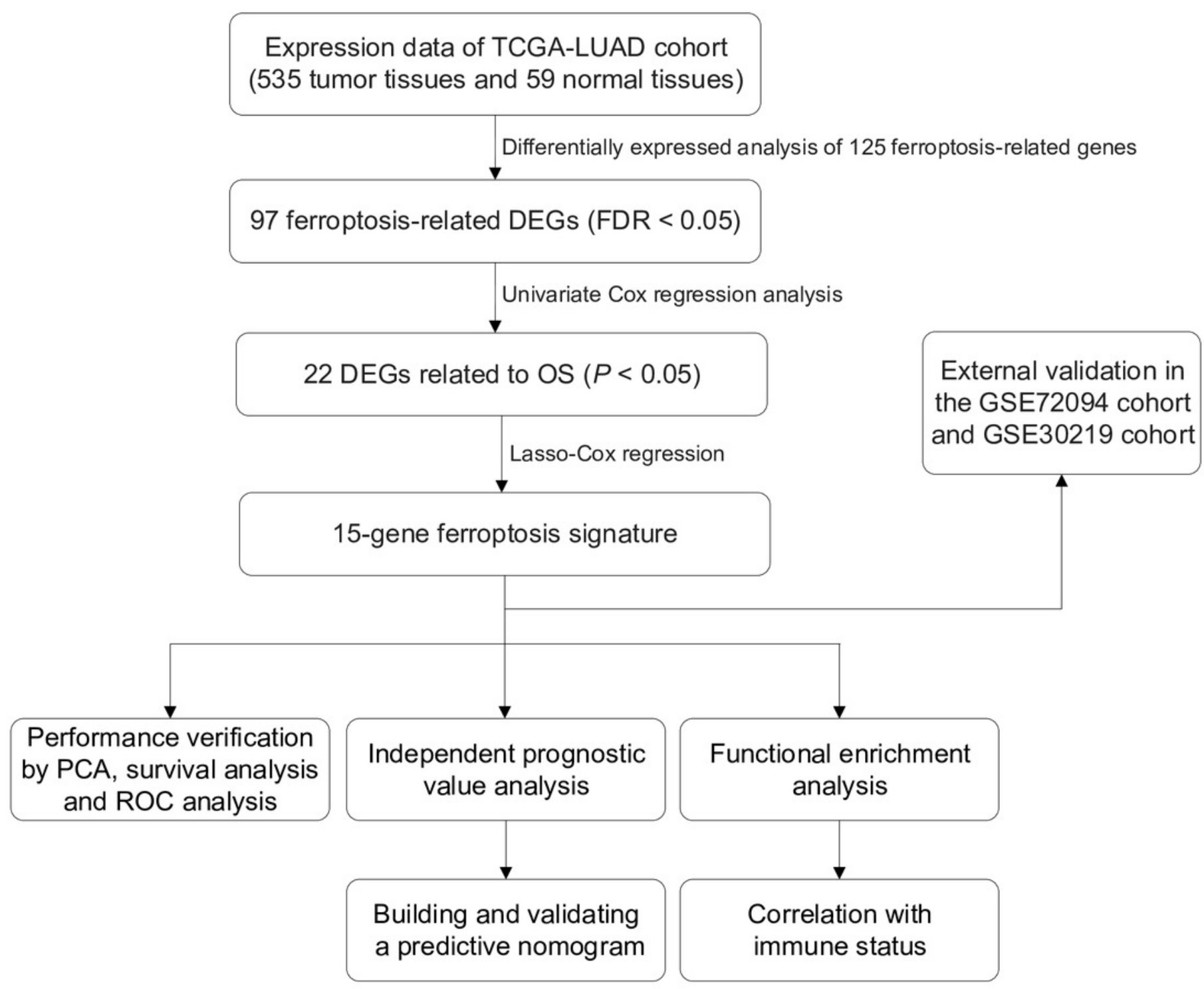




\section{Figure 2}

Heatmap and volcano plot of the ferroptosis-related DEGs.

(A) Heatmap of the top 20 upregulated and downregulated ferroptosis-related genes between normal and tumor tissues in the TCGA-LUAD cohort. (B) Volcano plot of the 97 ferroptosis-related DEGs in the TCGA-LUAD cohort. The top 5 up- and down-regulated ferroptosis-related DEGs were labeled in the volcano plot. FDR $<0.05$ was set as screening criteria. The blue, red and black dots represented the down-, up-regulated ferroptosisrelated DEGs and genes that were not satisfied the screening criteria, respectively. DEGs, differentially expressed genes; FDR, false discovery rate; TCGA, The Cancer Genome Atlas; LUAD, lung adenocarcinoma; N, normal tissues; T, tumor tissues.

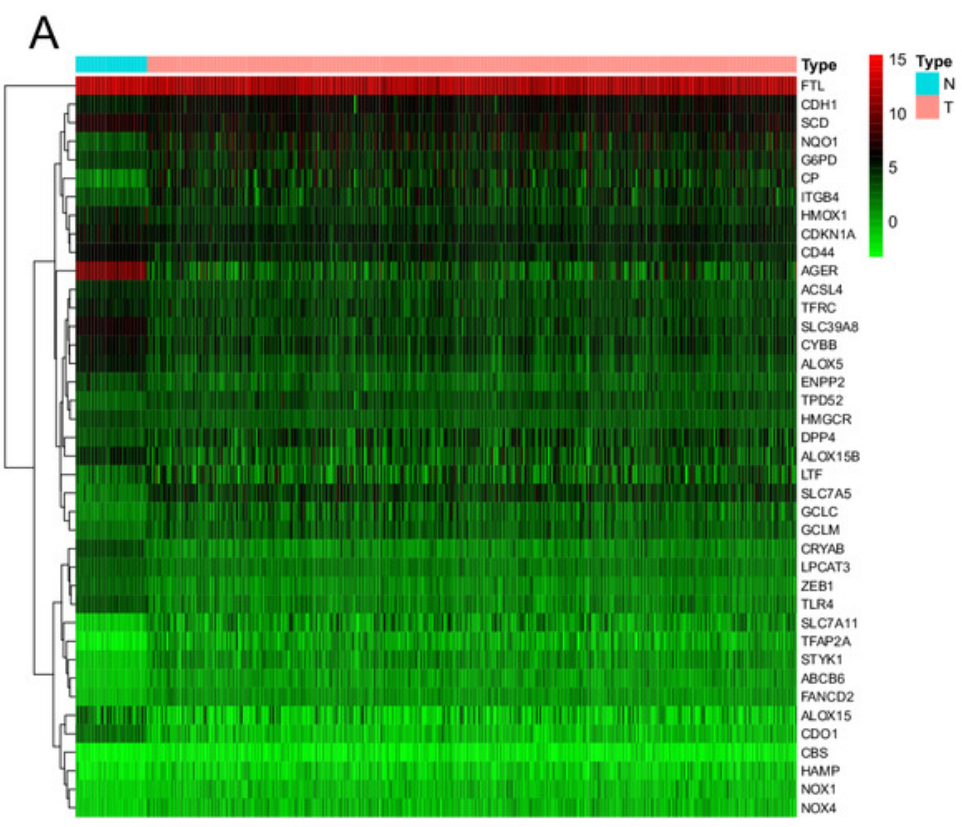

B

Volcano

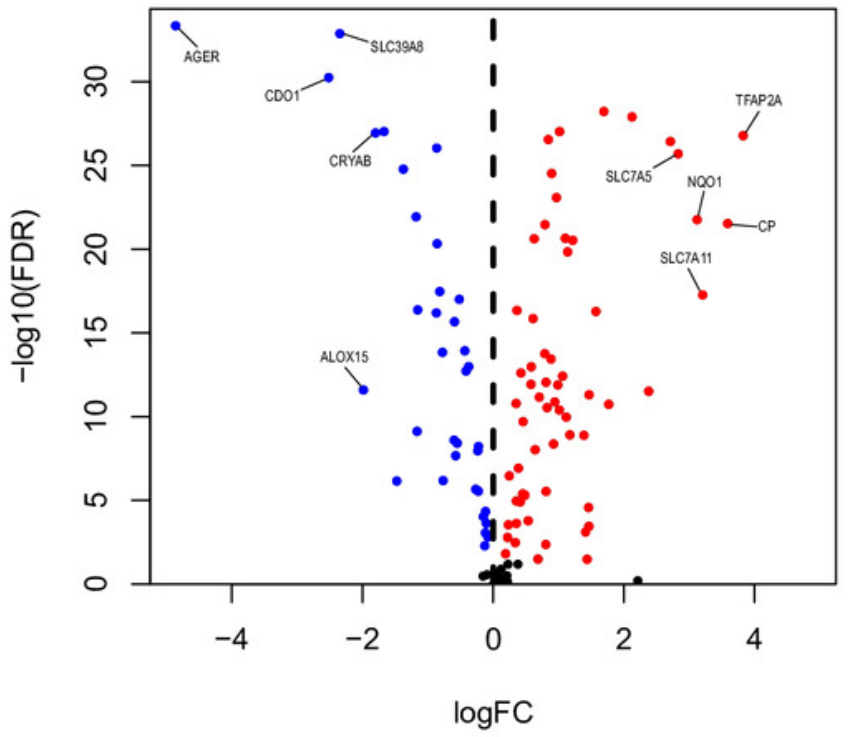


Figure 3

Forest map of 22 prognostic ferroptosis-related DEGs screened by univariate Cox regression.

DEGs, differentially expressed genes. Yellow: risk factors; blue: protective factors.

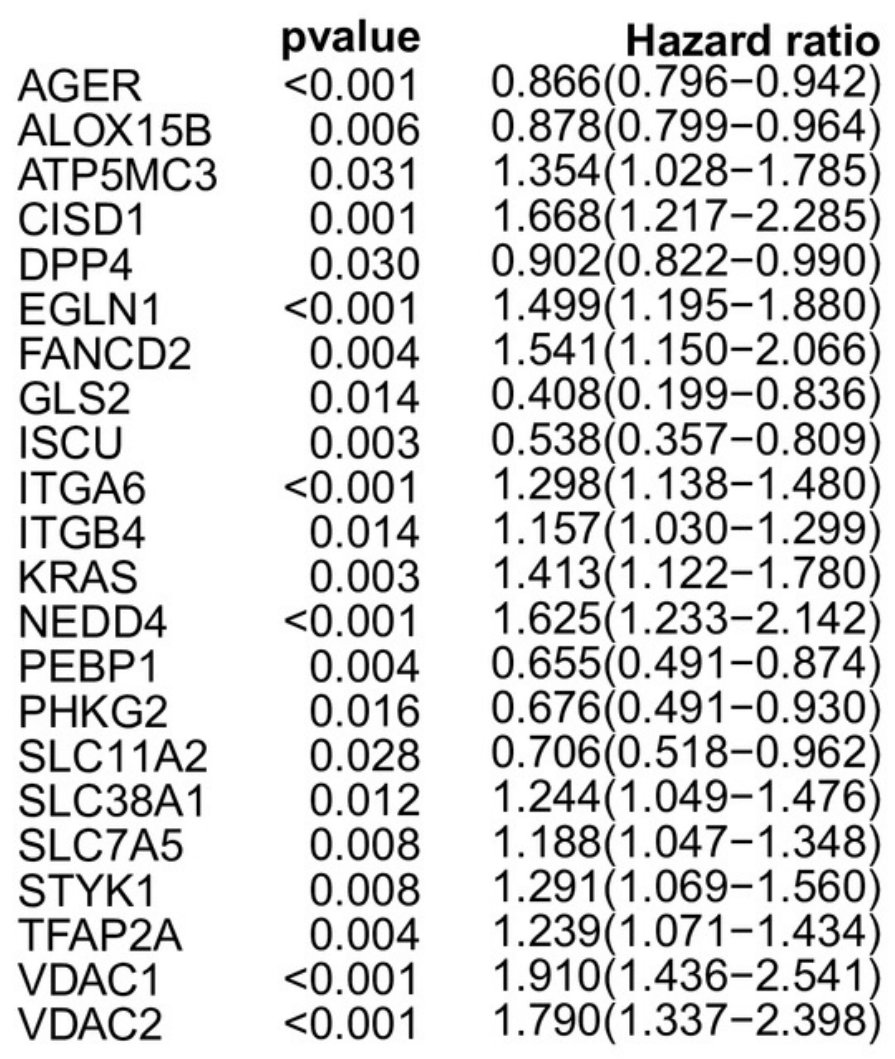

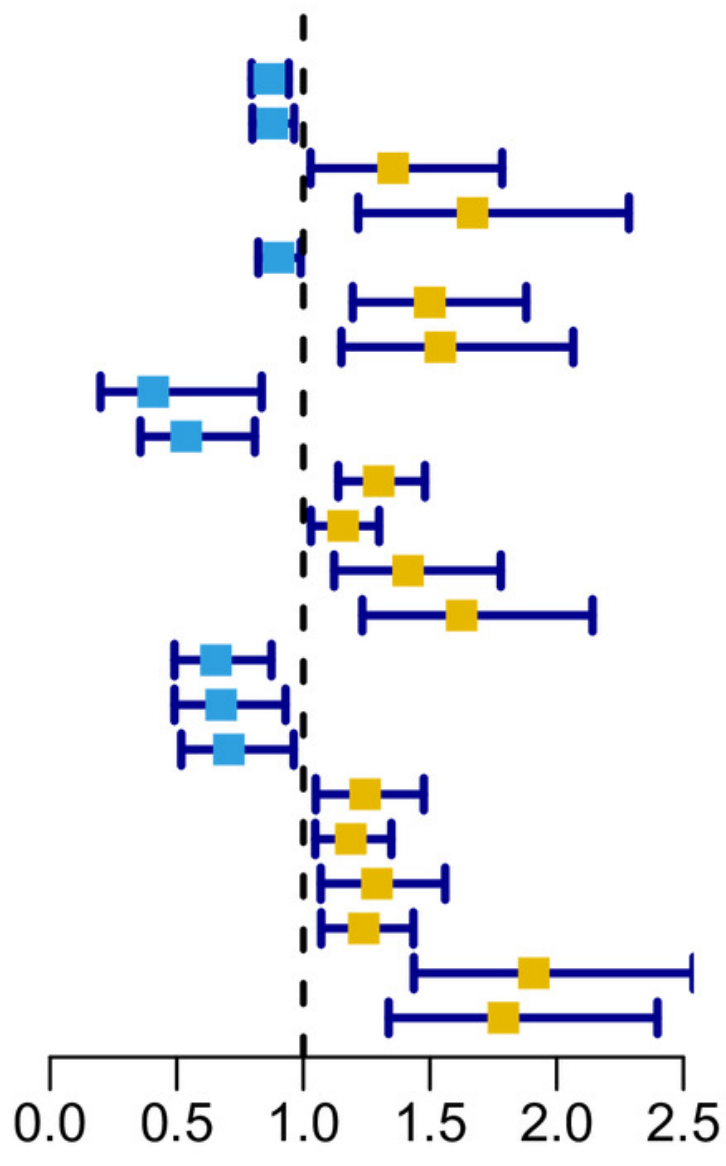

Hazard ratio 


\section{Figure 4}

Performance verification of the 15-gene ferroptosis signature in the TCGA cohort.

(A) PCA plot of patients in high-risk group (red) and low-risk group (navy blue). (B) Kaplan-

Meier curve of patients in high-risk group (red) and low-risk group (navy blue). (C) The

distribution of risk score of each patient. (D) The distribution of OS, survival status and risk score of each patient. Patients' OS was decreased with the increase of risk score. (E) Timedependent ROC curves of risk score and clinical characteristics. TCGA, The Cancer Genome Atlas; PCA, principal component analysis; OS, overall survival; AUC, area under curve; ROC, receiver operating characteristic. 
A

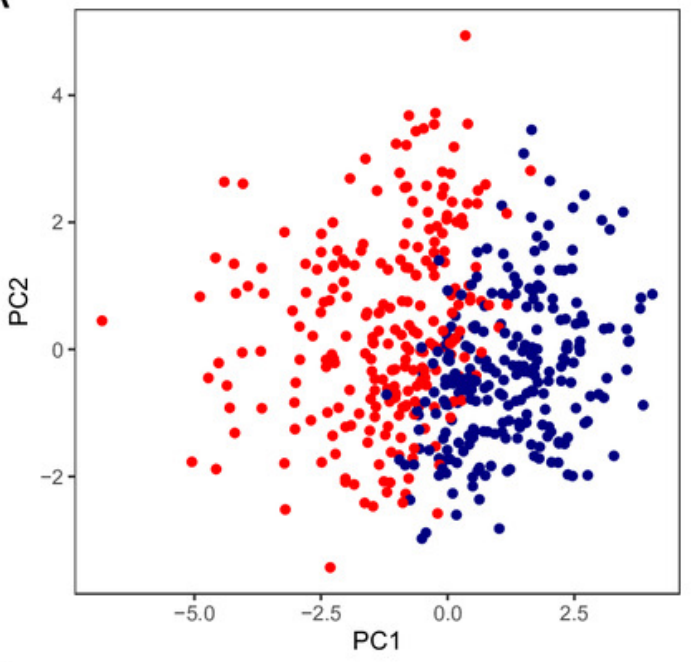

C

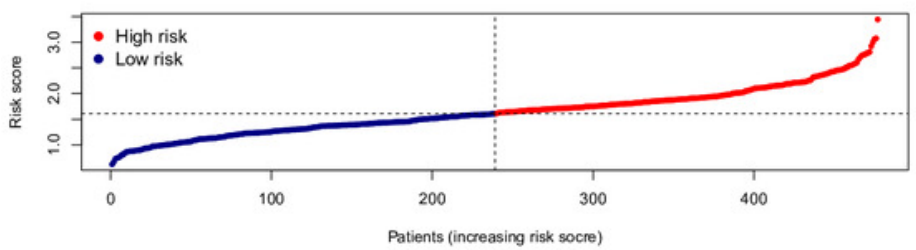

D

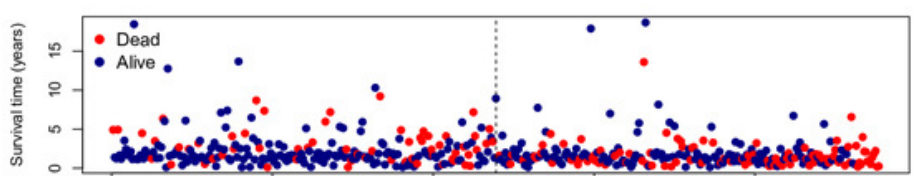

Risk

- high

- low
B

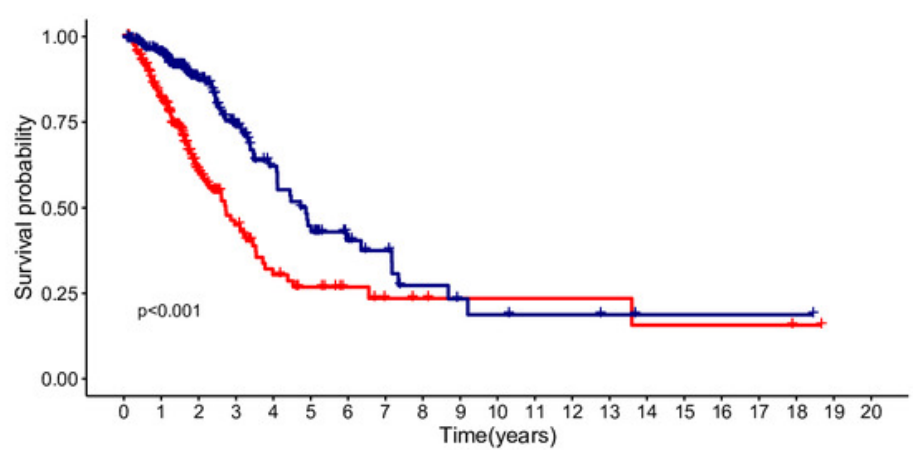

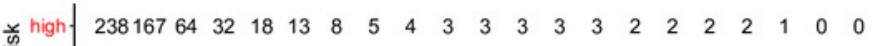

low. \begin{tabular}{ccccccccccccccccccccc}
239 & 196 & 95 & 58 & 36 & 25 & 16 & 12 & 7 & 5 & 4 & 3 & 3 & 2 & 1 & 1 & 1 & 1 & 1 & 0 & 0 \\
\hline 0 & 1 & 2 & 3 & 4 & 5 & 6 & $\overline{7}$ & $\mathbf{8}$ & $\mathbf{9}$ & 10 & 11 & 12 & 13 & 14 & 15 & 16 & 17 & 18 & 19 & 20
\end{tabular}

E

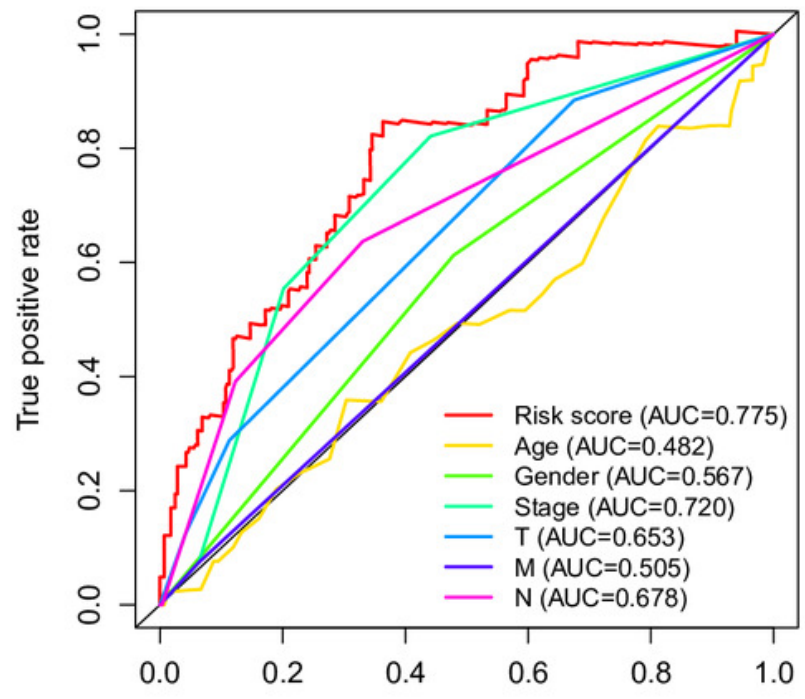

False positive rate 


\section{Figure 5}

Validation of the 15-gene ferroptosis signature in the GSE72094 cohort (A-E) and GSE30219 cohort (F-J).

(A, F) PCA plots of patients in high-risk group (red) and low-risk group (navy blue). (B, G) Kaplan-Meier curves of patients in high-risk group (red) and low-risk group (navy blue). (C, H) The distribution of risk score of each patient. (D, I) The distribution of OS, survival status and risk score of each patient. (E, J) Time- dependent ROC curves of risk score and clinical characteristics. PCA, principal component analysis; OS, overall survival; AUC, area under curve; ROC, receiver operating characteristic. 
A

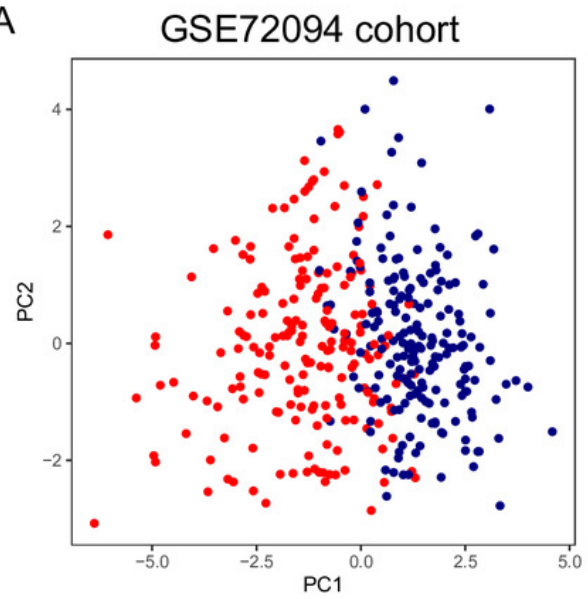

B

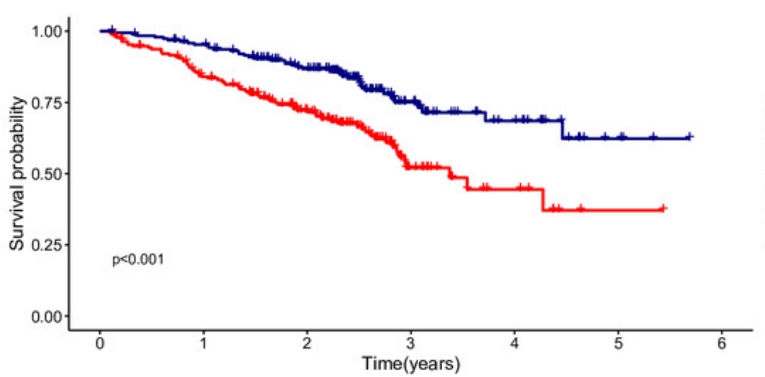

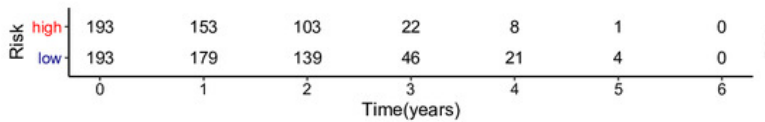

C

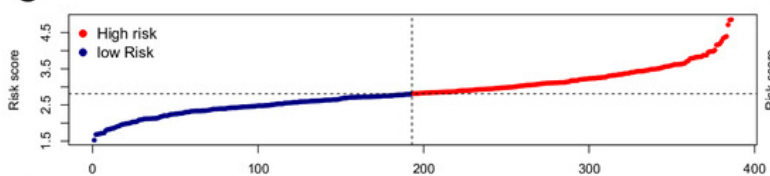

D

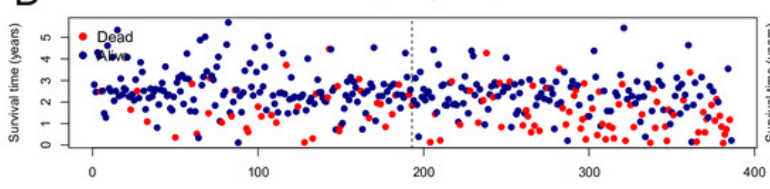

E

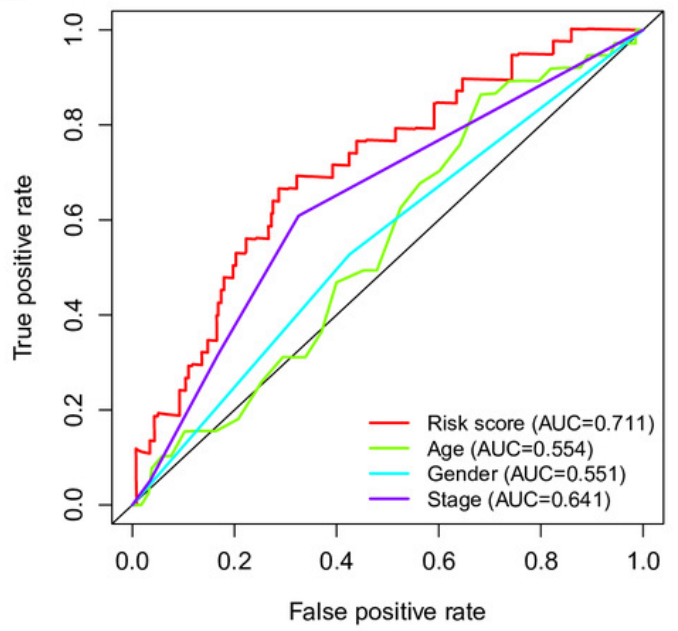

$\mathrm{F}$

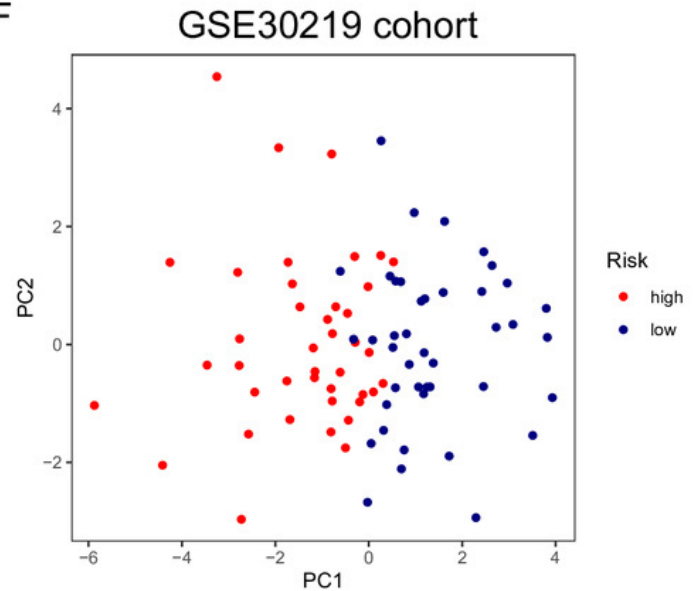

G

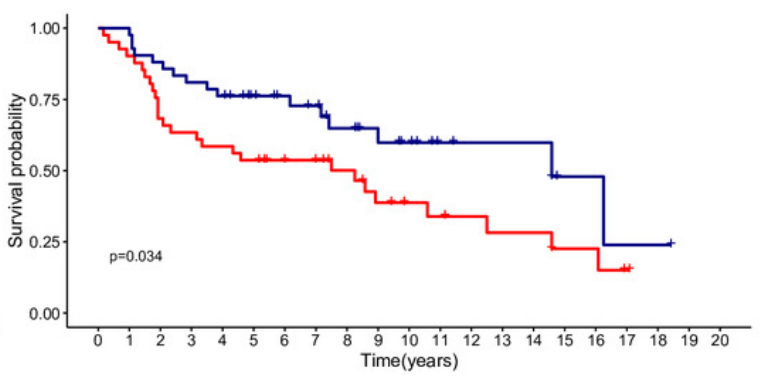

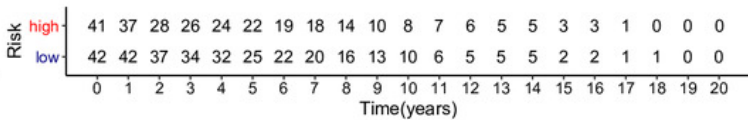

$\mathrm{H}$

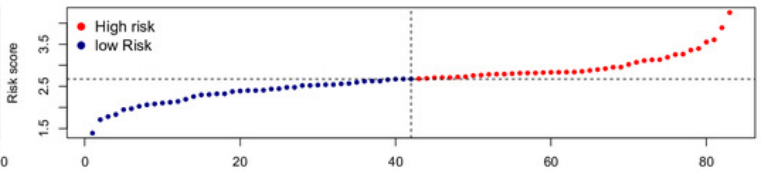

I

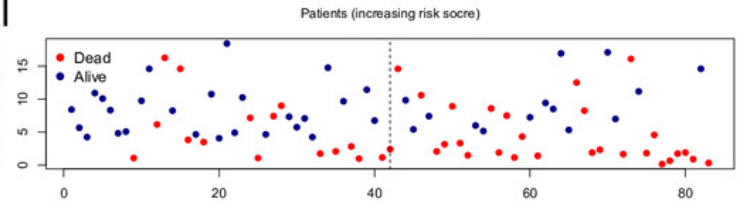

$\mathrm{J}$

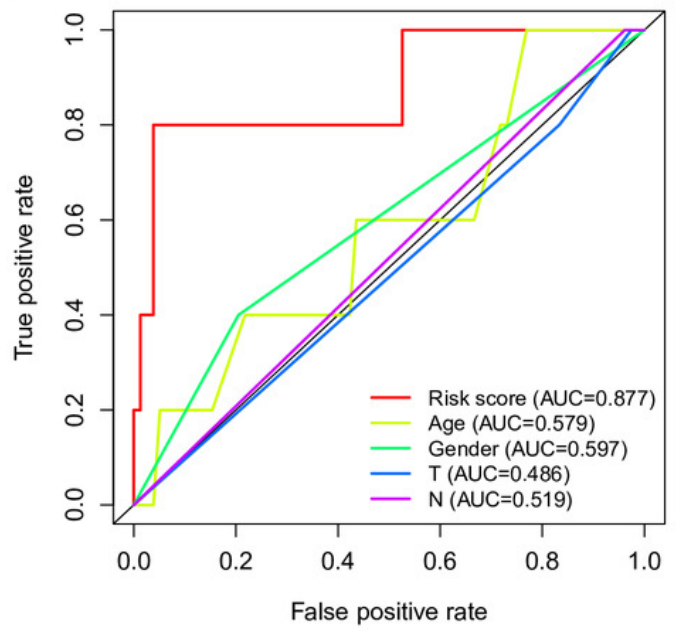




\section{Figure 6}

Stratification analyses of the TCGA cohort (A-B) and GSE72094 cohort (C-F).

(A, B) Kaplan-Meier curves of patients in high-risk group (red) and low-risk group (navy blue) stratified via stage I-II and stage III-IV in the TCGA cohort, respectively. (C, D) Kaplan-Meier curves of patients in high-risk group (red) and low-risk group (navy blue) stratified via female and male in the GSE72094 cohort, respectively. (E, F) Kaplan-Meier curves of patients in high-risk group (red) and low-risk group (navy blue) stratified via stage I-II and stage III-IV in the GSE72094 cohort. TCGA, The Cancer Genome Atlas. 


\section{TCGA cohort}

A

Patients with Stage I-II

Risk + high + low

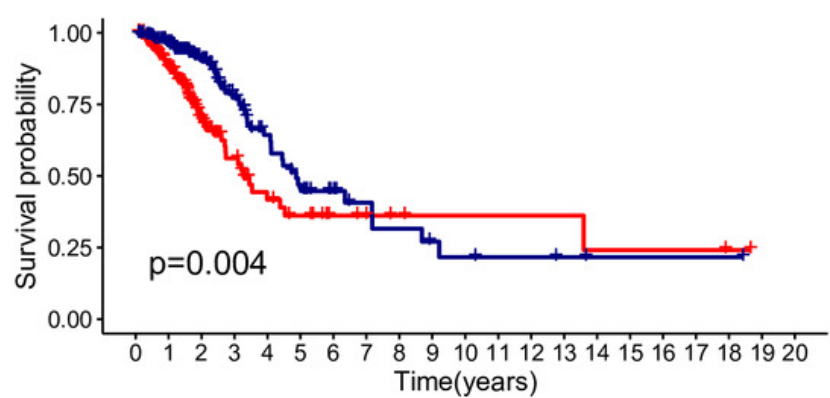

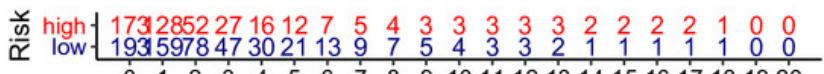

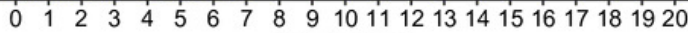
Time(years)

\section{GSE72094 cohort}

C

Patients with Female

Risk + high + low

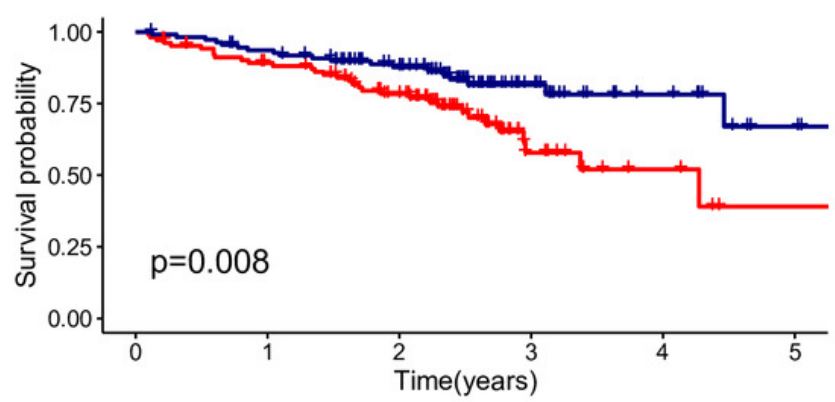

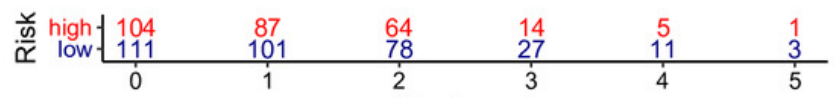

E

Patients with Stage I-II

Risk + high — low
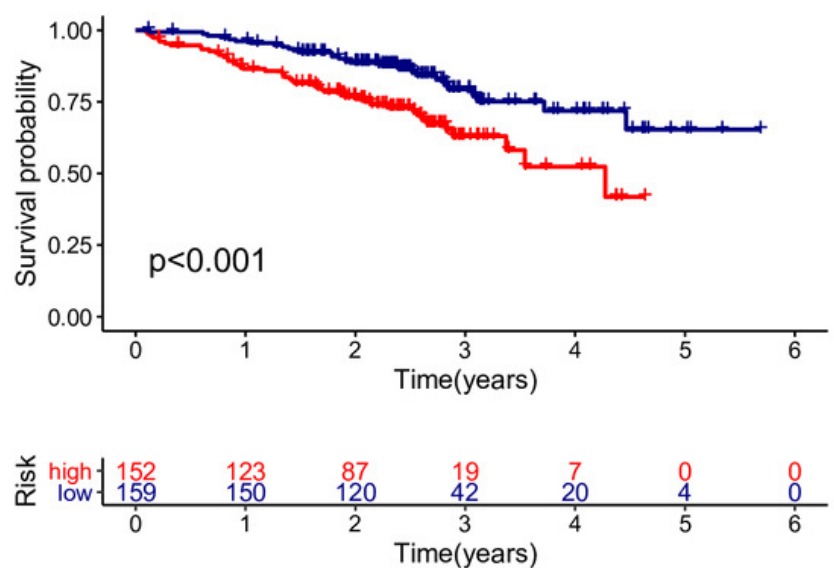

B

Patients with Stage III-IV
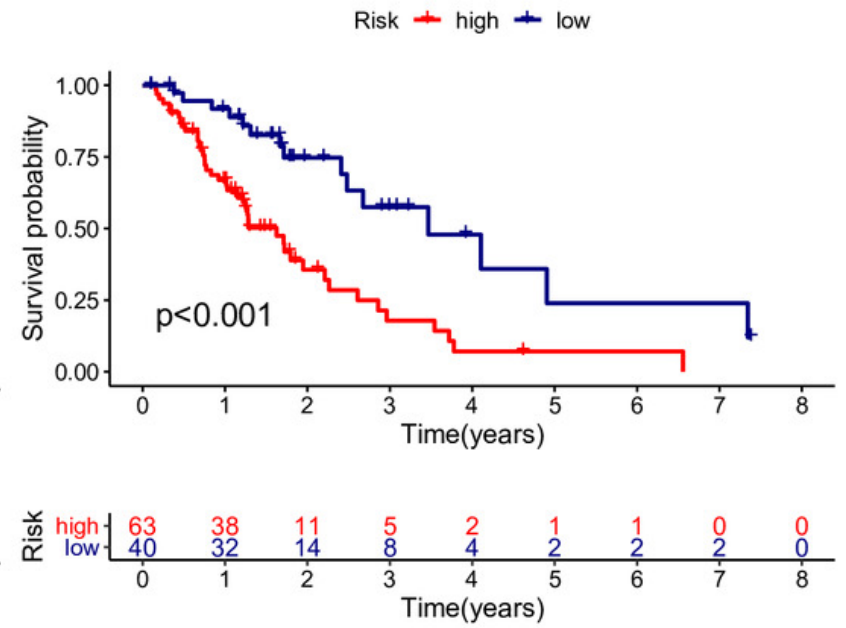

D

Patients with Male

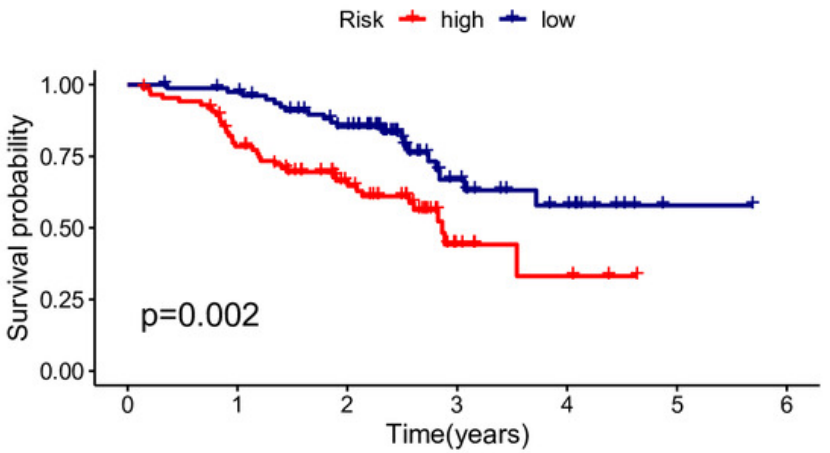

F

Patients with Stage III-IV

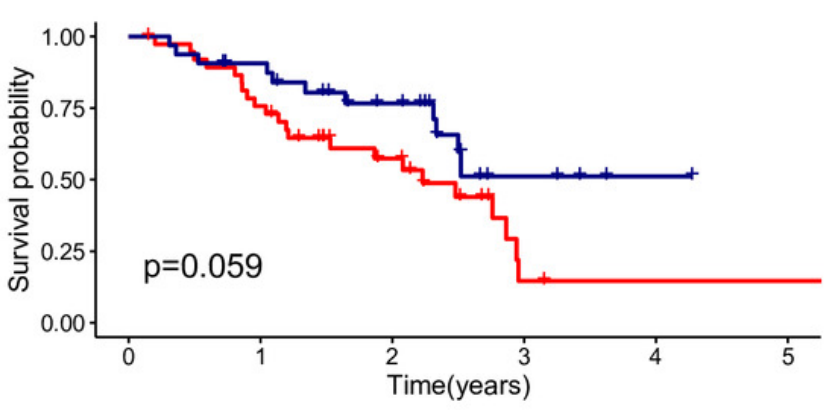

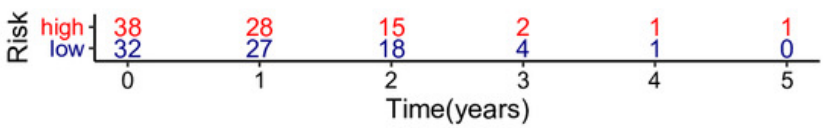




\section{Figure 7}

Construction and validation of the predictive nomogram.

(A) The nomogram for OS prediction at 1,2 and 3 years was constructed in the TCGA cohort.

(B) Kaplan-Meier curve of patients in high-risk group (red) and low-risk group (navy blue) in the TCGA cohort. (C) Kaplan-Meier curve of patients in high-risk group (red) and low-risk group (navy blue) in the GSE72094 cohort. (D, E) Calibration plots of nomogram for OS prediction at 1 year and 3 years in the TCGA cohort, respectively. $(F, G)$ Calibration plots of nomogram for OS prediction at 1 year and 3 years in the GSE72094 cohort, respectively. ( $H$, I) Time-dependent ROC curves to evaluate the predictive performance of nomogram at 1 year and 3 years in the TCGA cohort, respectively. (J, K) Time-dependent ROC curves to evaluate the predictive performance of nomogram at 1 year and 3 years in the GSE72094 cohort, respectively. In the calibration plot, the closer the red line (fitting line) and gray line (ideal line) are, the higher predictive accuracy of the model is. TCGA, The Cancer Genome Atlas; OS, overall survival; AUC, area under curve; ROC, receiver operating characteristic. 
A

Points

Stage

Riskscore

Total Points

1-year survival

2-year survival

3-year survival
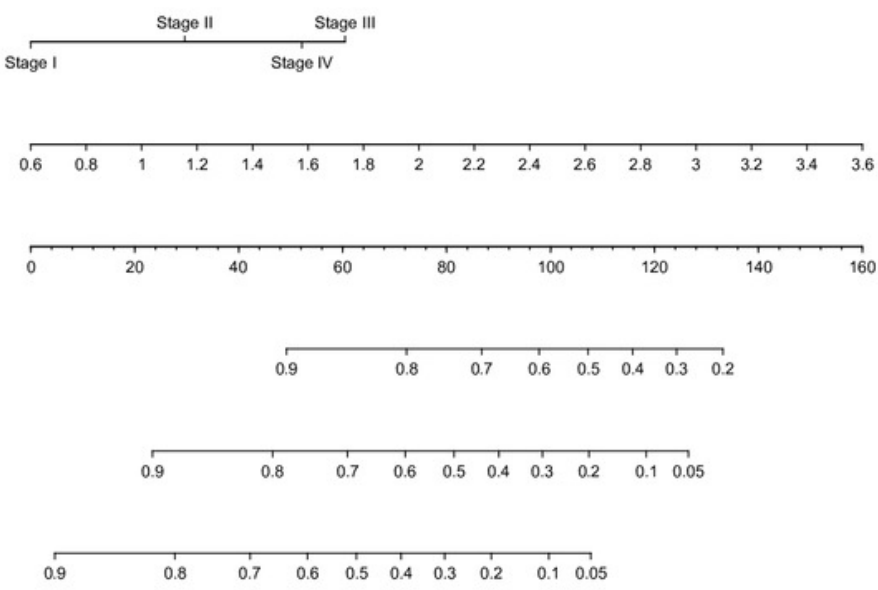

B

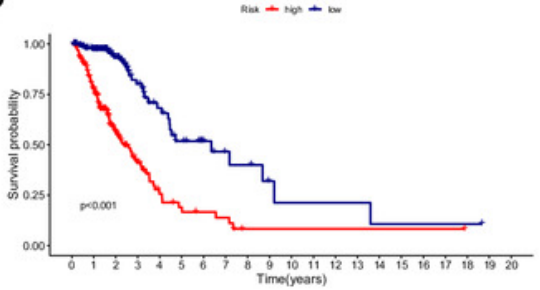

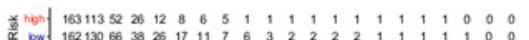

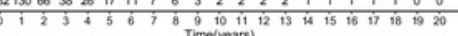

C
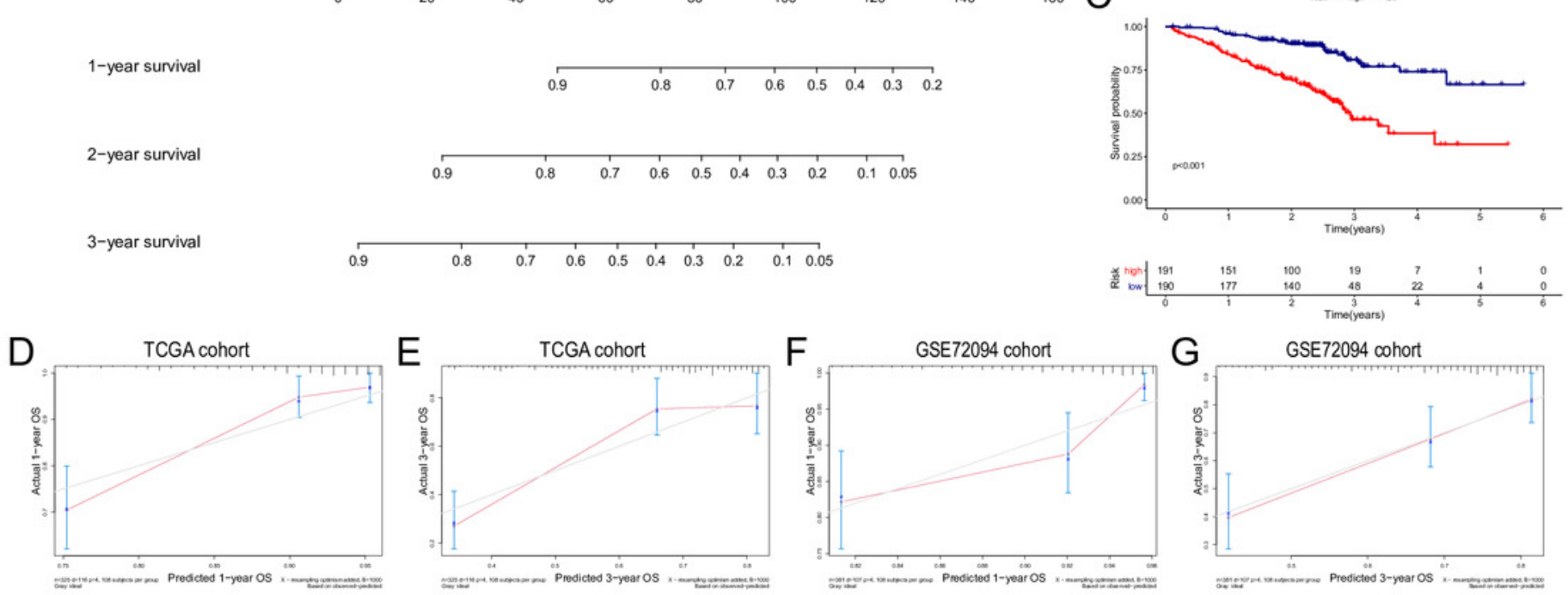

G GSE72094 cohort
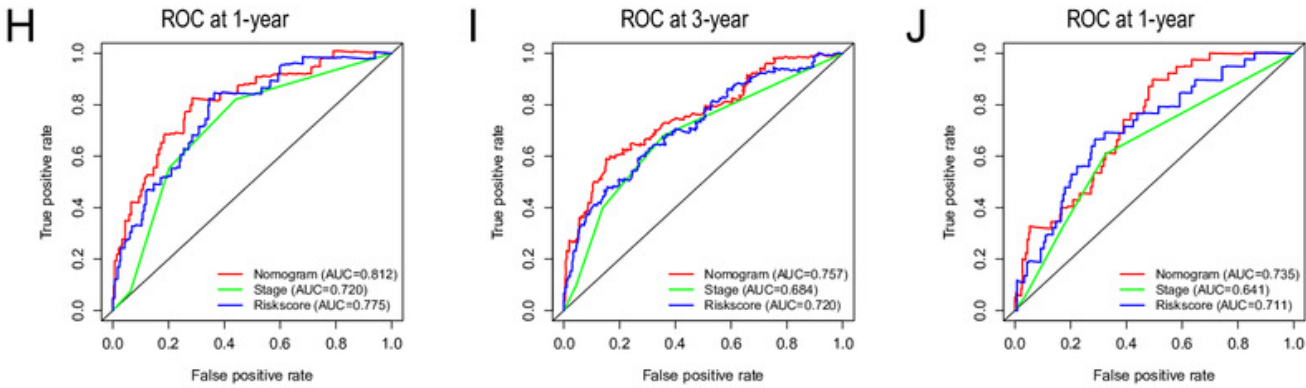

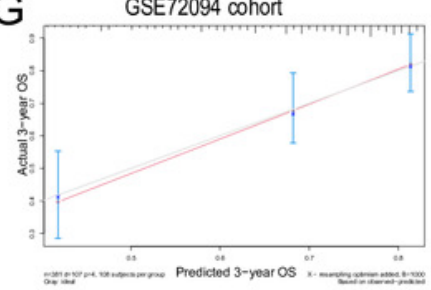

$\mathrm{K}$

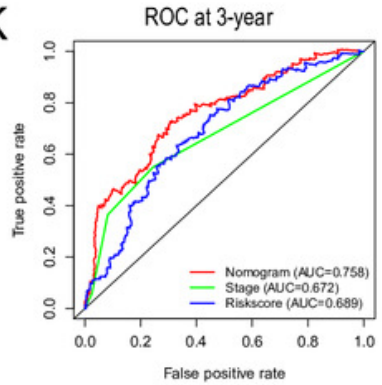




\section{Figure 8}

GO and KEGG pathway enrichment analyses.

(A) Representative GO pathways enriched in the DEGs between the high- and low-risk

groups. (B) The top 5 enriched KEGG pathways identified by GSEA in the high- and low-risk

groups. $|\log 2 \mathrm{FC}| \geq 1$ and $F D R<0.05$ were set as the criteria to screen DEGs between the highand low-risk groups. The pathways with a P value less than 0.05 were considered to be significantly enriched. DEGs, differentially expressed genes; GO, Gene Ontology; BP, Biological Process; CC, Cellular Component; MF, Molecular Function; KEGG, Kyoto Encyclopedia of Genes and Genomes; GSEA, Gene Set Enrichment Analysis. 


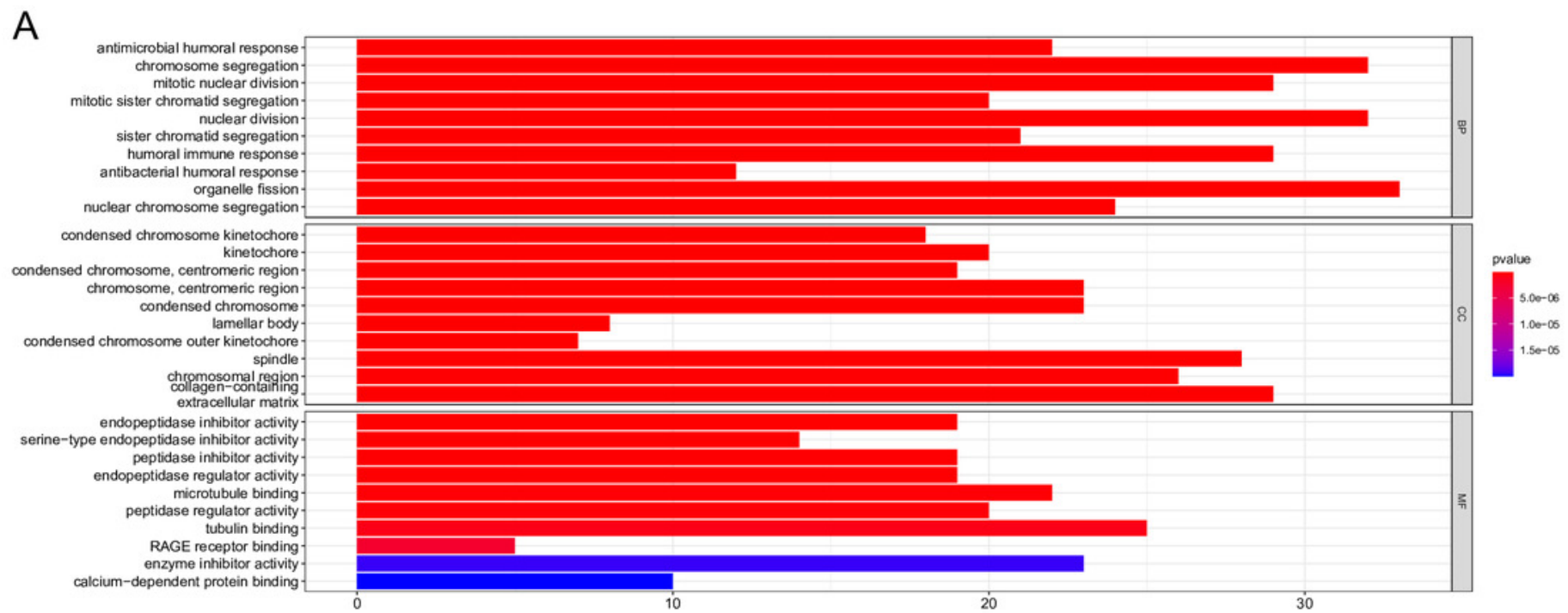

B

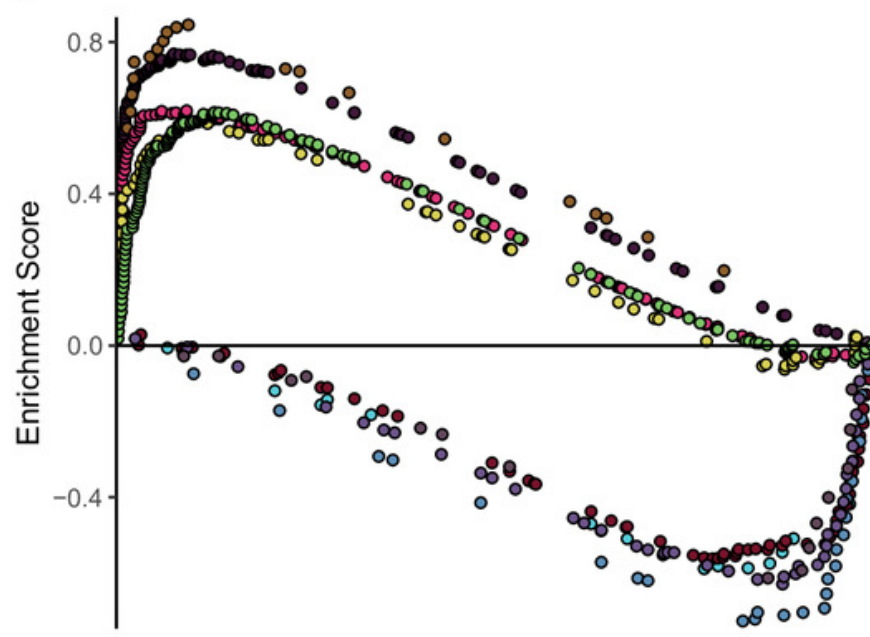

- KEGG_ALPHA_LINOLENIC_ACID_METABOLISM

- KEGG_ARACHIDONIC_ACID_METABOLISM

- KEGG_ASTHMA

- KEGG_CELL_CYCLE

- KEGG_HOMOLOGOUS_RECOMBINATION

- KEGG_INTESTINAL_IMMUNE_NETWORK_FOR_IGA_PRODUCTION

- KEGG_OOCYTE_MEIOSIS

- KEGG_P53_SIGNALING_PATHWAY

- KEGG_PRIMARY_BILE_ACID_BIOSYNTHESIS

- KEGG_UBIQUITIN_MEDIATED_PROTEOLYSIS

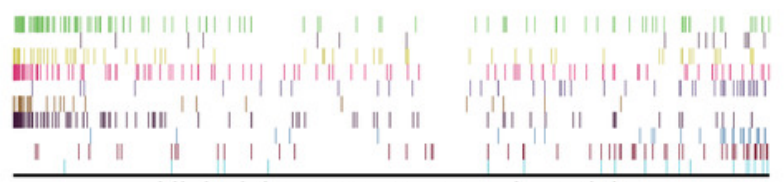

High risk<------------> Low risk 


\section{Figure 9}

Comparison of ssGSEA scores of different risk groups in the TCGA cohort (A, B), GSE72094 cohort (C, D) and GSE30219 cohort (E, F).

(A, C, E) Boxplots to display the enrichment scores of 16 immune cells of different risk groups in the TCGA cohort, GSE72094 cohort and GSE30219 cohort, respectively. (B, D, F) Boxplots to display the enrichment scores of 12 immune functions of different risk groups in the TCGA cohort and GSE72094 cohort and GSE30219 cohort, respectively. Adjusted P values were showed as: ns, not significant; *, $\mathrm{P}<0.05 ; * *, \mathrm{P}<0.01 ; * *, \mathrm{P}<0.001$. ssGSEA, single-sample gene set enrichment analysis; TCGA, The Cancer Genome Atlas; DC, Dendritic cells; APC, Antigen-presenting cells; Type II INF, IFN- $\gamma$. 


\section{TCGA cohort}

A

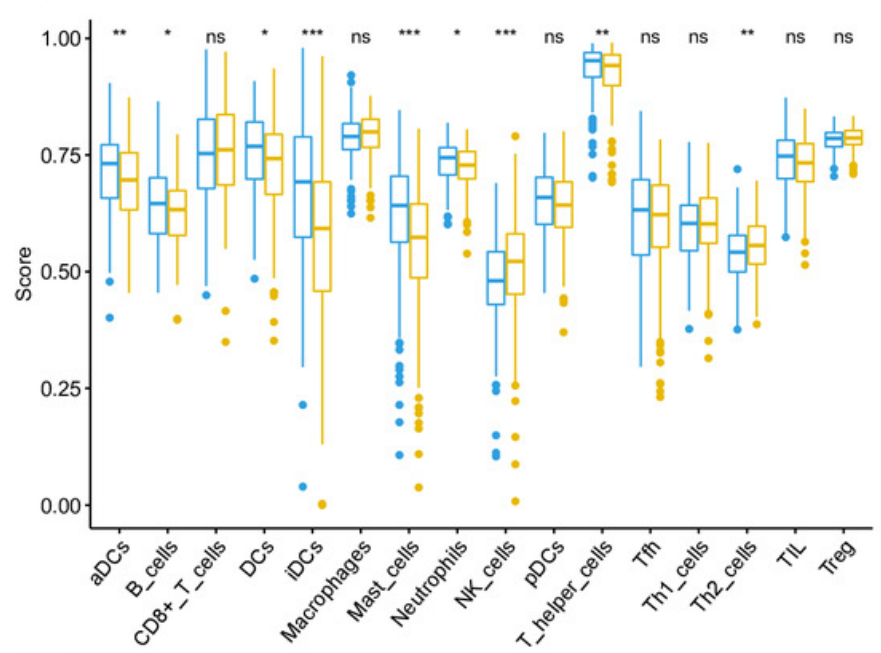

GSE72094 cohort

C

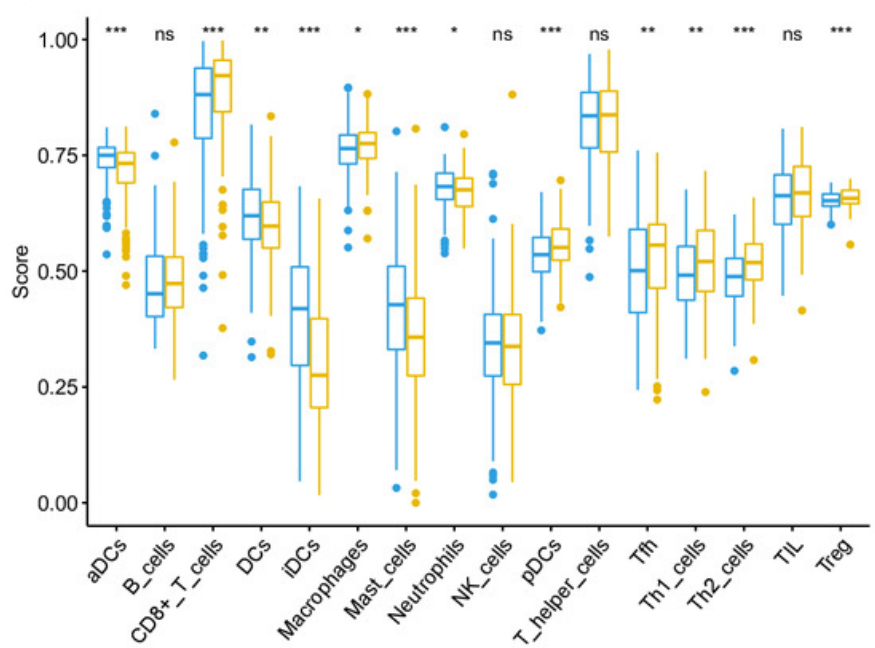

\section{GSE30219 cohort}

E

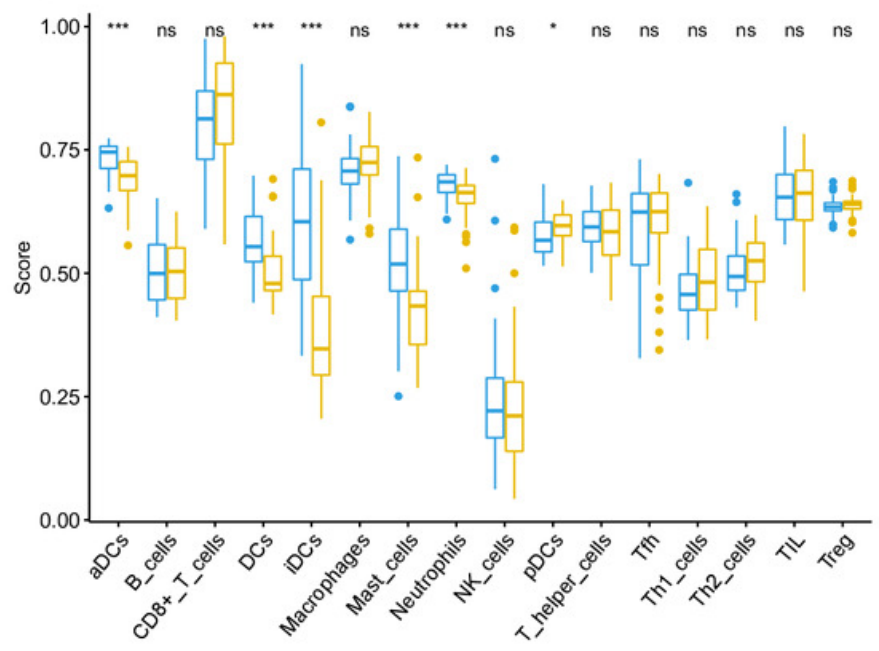

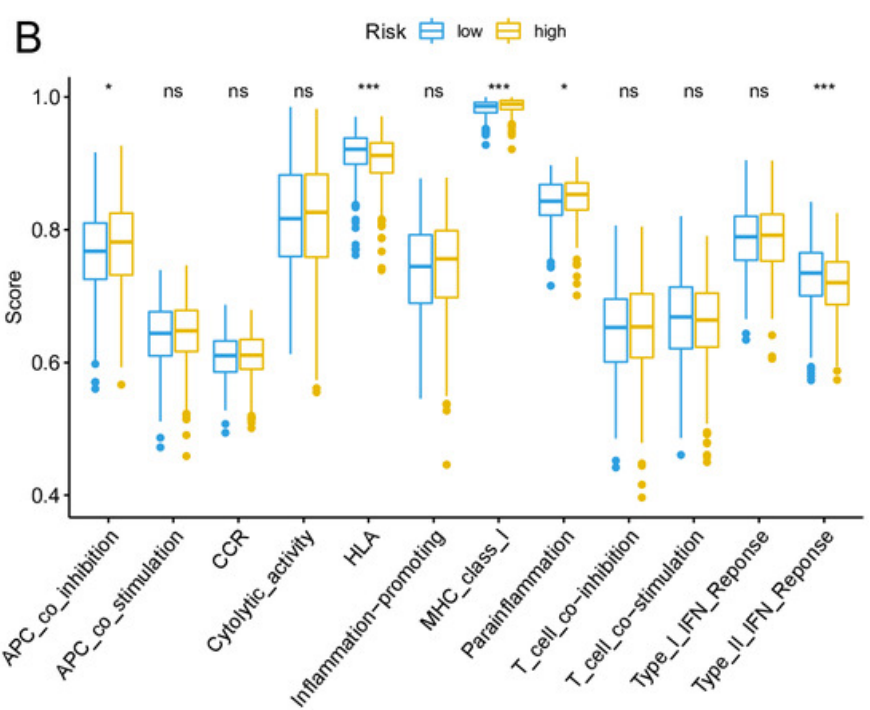

D Risk 户 low 官 high

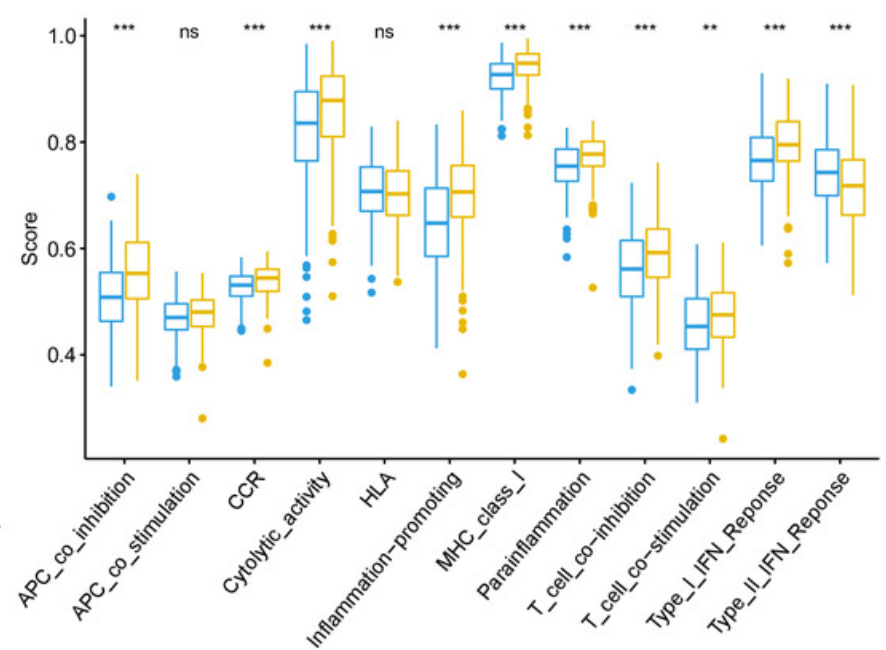

F Risk 帛 low 官 high

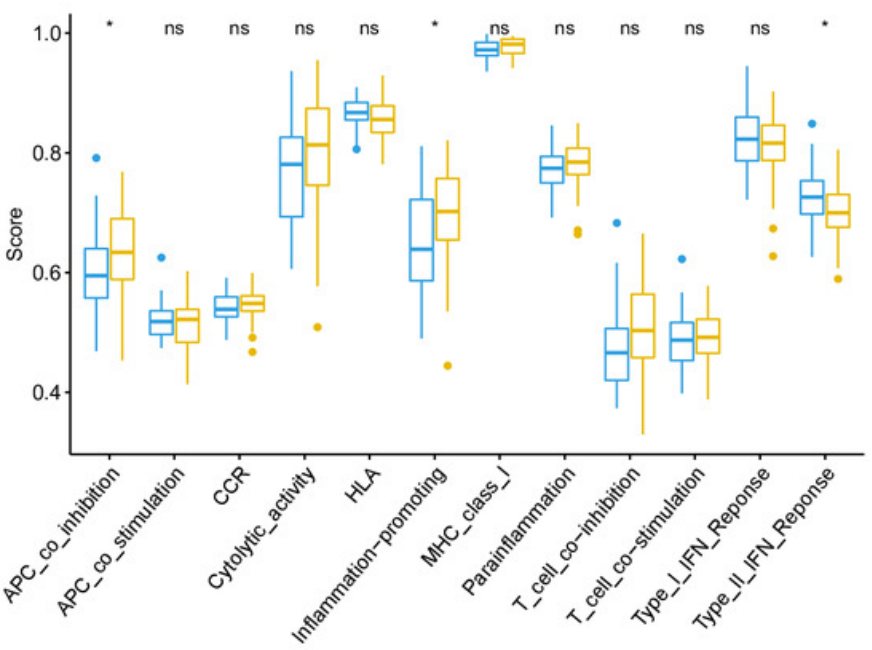




\section{Table 1 (on next page)}

Univariate and multivariate analysis of OS in the TCGA, GSE72094 and GSE30219 cohorts. 


\begin{tabular}{|c|c|c|c|c|c|c|}
\hline \multirow[b]{2}{*}{ Variables } & \multicolumn{3}{|c|}{ Univariate analysis } & \multicolumn{3}{|c|}{ Multivariate analysis } \\
\hline & $\mathrm{HR}$ & $95 \% \mathrm{Cl}$ & $P$ value & $\mathrm{HR}$ & $95 \% \mathrm{Cl}$ & $P$ value \\
\hline \multicolumn{7}{|c|}{ TCGA cohort } \\
\hline Age & 0.997 & $0.978-1.015$ & 0.718 & & & \\
\hline Gender & 1.000 & $0.694-1.441$ & 1.000 & & & \\
\hline Stage & 1.648 & $1.396-1.946$ & $<0.001$ & 1.591 & $1.064-2.380$ & 0.024 \\
\hline $\mathrm{T}$ & 1.600 & $1.285-1.994$ & $<0.001$ & 1.064 & $0.830-1.364$ & 0.626 \\
\hline $\mathrm{N}$ & 2.765 & $1.911-4.001$ & $<0.001$ & 1.316 & $0.723-2.397$ & 0.369 \\
\hline M & 1.748 & $0.959-3.187$ & 0.068 & & & \\
\hline Risk score & 3.670 & $2.521-5.341$ & $<0.001$ & 2.918 & $1.972-4.318$ & $<0.001$ \\
\hline \multicolumn{7}{|c|}{ GSE72094 cohort } \\
\hline Age & 1.010 & $0.990-1.030$ & 0.344 & & & \\
\hline Gender & 1.501 & $1.027-2.194$ & 0.036 & 1.547 & $1.050-2.280$ & 0.027 \\
\hline Stage & 1.628 & $1.358-1.951$ & $<0.001$ & 1.589 & $1.351-1.920$ & $<0.001$ \\
\hline Risk score & 2.607 & $1.918-3.544$ & $<0.001$ & 2.135 & $1.722-3.208$ & $<0.001$ \\
\hline \multicolumn{7}{|c|}{ GSE30219 cohort } \\
\hline Age & 1.031 & $0.996-1.067$ & 0.088 & & & \\
\hline Gender & 1.114 & $0.516-2.403$ & 0.784 & & & \\
\hline $\mathrm{T}$ & 1.463 & $0.856-2.499$ & 0.164 & & & \\
\hline$N$ & 1.247 & $0.299-5.202$ & 0.762 & & & \\
\hline Risk score & 2.881 & $1.516-5.474$ & 0.001 & 6.751 & $2.400-18.785$ & $<0.001$ \\
\hline
\end{tabular}

1 Abbreviations: TCGA, The Cancer Genome Atlas; HR, hazard ratio; $\mathrm{Cl}$, confidence interval. 


\section{Table 2 (on next page)}

Top 5 enriched KEGG pathways of the high-risk and low-risk groups analysed by GSEA. 


\begin{tabular}{|c|c|c|c|c|c|}
\hline Enriched pathways & Size & Es & NES & $\begin{array}{l}\text { NOM } \\
\mathrm{p} \text {-value }\end{array}$ & $\begin{array}{l}\text { FDR } \\
\text { q-value }\end{array}$ \\
\hline \multicolumn{6}{|l|}{ High-risk group } \\
\hline KEGG_CELL_CYCLE & 125 & 0.769 & 2.495 & $<0.001$ & $<0.001$ \\
\hline $\begin{array}{l}\text { KEGG_UBIQUITIN_MEDIATED_PROTEOLY- } \\
\text { SIS }\end{array}$ & 135 & 0.615 & 2.458 & $<0.001$ & $<0.001$ \\
\hline KEGG_OOCYTE_MEIOSIS & 113 & 0.619 & 2.342 & $<0.001$ & $<0.001$ \\
\hline KEGG_HOMOLOGOUS_RECOMBINATION & 28 & 0.845 & 2.291 & $<0.001$ & $<0.001$ \\
\hline $\begin{array}{l}\text { KEGG_P53_SIGNALING_PATHWAY } \\
\text { Low-risk group }\end{array}$ & 68 & 0.589 & 2.270 & $<0.001$ & $<0.001$ \\
\hline KEGG_ARACHIDONIC_ACID_METABOLISM & 58 & 0.571 & 1.988 & 0.002 & 0.103 \\
\hline $\begin{array}{l}\text { KEGG_PRIMARY_BILE_ACID_BIOSYNTHE- } \\
\text { SIS }\end{array}$ & 16 & 0.661 & 1.883 & $<0.001$ & 0.144 \\
\hline $\begin{array}{l}\text { KEGG_ALPHA_LINOLENIC_ACID_METABO- } \\
\text { LISM }\end{array}$ & 19 & 0.621 & 1.823 & 0.006 & 0.156 \\
\hline KEGG_ASTHMA & 28 & 0.745 & 1.820 & 0.017 & 0.119 \\
\hline $\begin{array}{l}\text { KEGG_INTESTINAL_IMMUNE_NETWORK_ } \\
\text { FOR_IGA_PRODUCTION }\end{array}$ & 46 & 0.650 & 1.740 & 0.040 & 0.177 \\
\hline
\end{tabular}

1 Abbreviations: ES, enrichment score; NES, normalized enrichment score; NOM p-value, nominal $p$ 2 value; FDR q-valve, false discovery rate. 


\section{Table 3(on next page)}

Modulatory effect of the signature genes on ferroptosis. 


\begin{tabular}{|c|c|c|c|}
\hline $\begin{array}{l}\text { Gene } \\
\text { symbol }\end{array}$ & Protein & Modulatory effect on ferroptosis & References \\
\hline AGER & $\begin{array}{l}\text { advanced glycosylation } \\
\text { end product-specific } \\
\text { receptor }\end{array}$ & $\begin{array}{l}\text { depletion attenuates the autophagy- } \\
\text { dependent ferroptosis }\end{array}$ & $\underline{\text { Wen et al. }}$ \\
\hline CISD1 & $\begin{array}{l}\text { CDGSH iron-sulfur domain- } \\
\text { containing protein } 1\end{array}$ & $\begin{array}{l}\text { down-expression contributes to } \\
\text { ferroptosis by iron-mediated } \\
\text { intramitochondrial lipid peroxidation }\end{array}$ & $\frac{\text { Yuan et al. }}{\underline{2016}}$ \\
\hline DPP4 & dipeptidyl peptidase 4 & $\begin{array}{l}\text { overexpression protects cell from } \\
\text { ferroptosis by inhibiting lipid peroxidation }\end{array}$ & $\underline{\underline{\text { Xie et al. }}}$ \\
\hline EGLN1 & egl nine homolog 1 & $\begin{array}{l}\text { overexpression inhibits ferroptosis by the } \\
\text { induction of LSH }\end{array}$ & $\begin{array}{l}\text { Jiang et al. } \\
\underline{2017}\end{array}$ \\
\hline FANCD2 & $\begin{array}{l}\text { Fanconi anemia group D2 } \\
\text { protein }\end{array}$ & $\begin{array}{l}\text { knockout promotes ferroptosis though } \\
\text { increasing iron accumulation and lipid } \\
\text { peroxidation }\end{array}$ & $\begin{array}{l}\text { Song et al. } \\
\underline{2016}\end{array}$ \\
\hline GLS2 & glutaminase liver isoform & $\begin{array}{l}\text { upregulation promotes ferroptosis in } \\
\text { gastric cancer }\end{array}$ & $\underline{\underline{\text { Niu et al. }}}$ \\
\hline ISCU & $\begin{array}{l}\text { iron-sulfur cluster assembly } \\
\text { enzyme ISCU }\end{array}$ & $\begin{array}{l}\text { overexpression attenuates ferroptosis by } \\
\text { increasing the level of GSH }\end{array}$ & $\begin{array}{l}\text { Du et al. } \\
\underline{2019}\end{array}$ \\
\hline ITGA6 & integrin alpha-6 & $\begin{array}{l}\text { overexpression inhibits ferroptosis by } \\
\text { suppressing the expression of ACSL4 }\end{array}$ & $\begin{array}{l}\text { Brown et al. } \\
\underline{2017}\end{array}$ \\
\hline ITGB4 & integrin beta-4 & $\begin{array}{l}\text { overexpression inhibits ferroptosis by } \\
\text { suppressing the expression of ACSL4 }\end{array}$ & $\begin{array}{l}\text { Brown et al. } \\
\underline{2017}\end{array}$ \\
\hline KRAS & GTPase KRas & $\begin{array}{l}\text { inhibits ferroptosis by upregulating the } \\
\text { expression of SLC7A11 }\end{array}$ & $\underline{\underline{\text { Hu et al. }}}$ \\
\hline NEDD4 & $\begin{array}{l}\text { E3 ubiquitin-protein ligase } \\
\text { NEDD4 }\end{array}$ & $\begin{array}{l}\text { upregulation inhibits ferroptosis induced } \\
\text { by erastin through the degradation of } \\
\text { VDAC2/3 }\end{array}$ & $\frac{\text { Yang et al. }}{\underline{2020}}$ \\
\hline PEBP1 & $\begin{array}{l}\text { phosphatidylethanolamine- } \\
\text { binding protein } 1\end{array}$ & $\begin{array}{l}\text { overexpression increases sensitivity to } \\
\text { ferroptosis in HAEC and HT22 cells }\end{array}$ & $\begin{array}{l}\text { Wenzel et } \\
\text { al. } 2017\end{array}$ \\
\hline SLC11A2 & $\begin{array}{l}\text { natural resistance- } \\
\text { associated macrophage } \\
\text { protein } 2\end{array}$ & $\begin{array}{l}\text { upregulation contributes to ferroptosis by } \\
\text { increasing iron uptake }\end{array}$ & $\begin{array}{l}\text { Song et al. } \\
\underline{2020}\end{array}$ \\
\hline TFAP2A & $\begin{array}{l}\text { transcription factor AP-2- } \\
\text { alpha }\end{array}$ & $\begin{array}{l}\text { negatively regulates ferroptosis by } \\
\text { increasing the expression of NRF2 }\end{array}$ & $\begin{array}{l}\text { Huang et al. } \\
\underline{2020}\end{array}$ \\
\hline VDAC1 & $\begin{array}{l}\text { voltage-dependent anion- } \\
\text { selective channel protein } 1\end{array}$ & $\begin{array}{l}\text { inhibition alleviates ferroptosis by } \\
\text { decreasing the ROS level in mitochondria }\end{array}$ & $\begin{array}{l}\text { Nagakanna } \\
\text { n et al. } 2019\end{array}$ \\
\hline
\end{tabular}

